\title{
On Monotone Strategy Equilibria in Simultaneous Auctions for Complementary Goods *
}

\author{
Matthew Gentry ${ }^{\dagger} \quad$ Tatiana Komarova ${ }^{\ddagger} \quad$ Pasquale Schiraldi $^{\S}$ \\ Wiroy Shin
}

August 12, 2019

\begin{abstract}
We explore existence and properties of equilibrium when $N \geq 2$ bidders compete for $L \geq 2$ objects via simultaneous but separate auctions. Bidders have private combinatorial valuations over all sets of objects they could win, and objects are complements in the sense that these valuations are supermodular in the set of objects won. We provide a novel partial order on types under which best replies are monotone, and demonstrate that Bayesian Nash equilibria which are monotone with respect to this partial order exist on any finite bid lattice. We apply this result to show existence of monotone Bayesian Nash equilibria in continuous bid spaces when a single global bidder competes for $L$ objects against many local bidders who bid for single objects only. We then consider monotone equilibrium with endogenous tiebreaking building on Jackson, Simon, Swinkels and Zame (2002), and demonstrate that these exist in general. These existence results apply to many auction formats, including first-price, second-price, and all-pay.
\end{abstract}

*This article incorporates materials from the prior working papers "Simultaneous Auctions for Complementary Goods" by W. Shin and "On Monotone Strategy Equilibria in Simultaneous Auctions for Complementary Goods" by M. Gentry, T. Komarova, and P. Schiraldi. We are grateful to Edward Green, Vijay Krishna, Roger Myerson and Balazs Szentes for their comments and insight.

${ }^{\dagger}$ Florida State University, mgentry@fsu.edu

†London School of Economics, t.komarova@lse.ac.uk

$\S$ London School of Economics and CEPR, p.schiraldi@lse.ac.uk

ฯKorea Institute for Industrial Economics and Trade, wshin@kiet.re.kr

$\sharp$ M. Gentry, T.Komarova and P. Schiraldi acknowledge the ESRC for financial support.

$\sharp$ W. Shin received financial support from the Human Capital Foundation to the Pennsylvania 


\section{Introduction}

Simultaneous bidding for multiple objects is a commonly occurring phenomenon in many real-world auction markets, but surprisingly little is known about the properties of equilibria in games involving simultaneous auctions when bidder payoffs are non-additive. ${ }^{1}$ For example, when auctioning drilling rights in the US Outer Continental Shelf, the US Minerals Management Service typically offers (and bidders typically bid on) a large number of drilling tracts simultaneously. Prior empirical work (e.g. Hendricks and Porter, 1988, Hendricks, Pinkse and Porter, 2003) suggests that economically important complementarities may exist between tracts in close proximity. Yet little is presently known - either theoretically or empirically - about how such synergies might affect equilibrium behavior in such markets. ${ }^{2}$

This paper analyzes equilibrium within a class of mechanisms we refer to as simultaneous standard auctions for complementary goods. In this setting, a collection of $L \geq 2$ objects are offered for sale to a set of $N \geq 2$ bidders. Bidders have independent private valuations over combinations of objects, where objects are complements in the sense that bidders' valuations are supermodular in sets of objects won. Auctions are simultaneous in the sense that bidders may bid on each object individually but may not submit contingent or combinatorial bids, and standard in the sense that each object $l$ is allocated to the highest bidder in auction $l$ and payments in auction

\section{State University.}

${ }^{1}$ Examples of markets involving simultaneous bidding include highway procurement in many US states (e.g. Krasnokutskaya [2011], Somaini [2013], Groeger [2014] among others), recycling services in Japan (Kawai, 2010), cleaning services in Sweden (Lunander and Lundberg, 2013), oil and drilling rights in the US Outer Continental Shelf (Hendricks and Porter, 1988, Hendricks, Pinkse and Porter, 2003), and to a lesser extent US Forest Service timber harvesting (Athey, Levin and Siera, 2011, among many others).

${ }^{2}$ Notable exceptions are Fox and Bajari (2013), who estimate the deterministic component of bidder valuations in FCC simultaneous ascending spectrum auctions, and Gentry, Komarova and Schiraldi (2017), who empirically study simultaneous bidding in Michigan Department of Transportation highway procurement auctions. 
$l$ depend only on bids in auction $l$. So long as all auctions are standard, auctions for different objects may have different formats. For simplicity, we frame discussion in terms of a single auctioneer, although this is inessential for our results.

The simultaneous standard auction game raises a number of significant theoretical challenges. Even assuming independent private types, each bidder's preference structure could in principle be as complex as a complete $\left(2^{L}-1\right)$-dimensional set of valuations assigned by that bidder to each of the $2^{L}-1$ possible non-empty subsets of objects. Meanwhile, the simultaneous standard auction permits bidders to submit (at most) $L$ individual bids on the $L$ objects being sold. Furthermore, as usual in auctions, payoffs in the resulting game may be discontinuous in bids. The end result is a discontinuous Bayesian game with high-dimensional types for which even basic properties - such as existence of Bayesian Nash equilibrium - are challenging to establish in general.

Section 2 introduces the model and describes what we mean by a standard auction. The framework of standard auctions includes many auction formats, such as first-price, second-price, and all-pay. Section 3 introduces a partial order on bidder types characterized by a finite number of linear inequalities on marginal valuations. These inequalities define a cone (with a nonempty interior) strictly contained in the first-orthant cone of the $\left(2^{L}-1\right)$-dimensional type space, which is the cone describing the usual coordinatewise order. We show that even the (strong) assumption of supermodular valuations is insufficient to ensure monotonicity of best replies with respect to the usual coordinatewise order on types - in fact, a strict coordinatewise increase in type can induce a strict coordinatewise decrease in best-reply bids. Our stronger partial order, however, is sufficient for monotonicity in that each bidder $i$ has an interim best reply such that an increase in $i$ 's type with respect to our partial 
order will imply an increase in $i$ 's bids with respect to the usual coordinatewise order.

Equipped with this preliminary result, Section 4 builds on the methodology of Athey (2001), McAdams (2003) and Reny (2011) to establish existence, in any simultaneous standard auction, of pure strategy equilibria on finite bid spaces which are monotone in the sense above. This result turns on one additional condition, which clarifies the relationship between the partial order cone and the support of bidder's joint distribution of valuations. Namely, we show that this relationship is "sufficiently rich" if this distribution is absolutely continuous with respect to the Lebesgue measure in the $\left(2^{L}-1\right)$-dimensional space and the support of this distribution is regular enough. While the existence of a pure strategy Bayesian Nash Equilibrium follows from Milgrom and Weber (1985), the monotone characterization of the equilibria under a suitable partial order on types is novel in this setting.

We then proceed to consider continuous bidding spaces. First, in Section 5.1, we consider a special case similar in spirit to Krishna and Rosenthal (1996), in which a single global bidder bids in simultaneous first-price auctions for $L$ objects against a collection of local bidders who bid for single objects only. ${ }^{3}$ Building on proof techniques in Reny (2011), we show existence of a pure strategy Bayesian Nash equilibrium which is monotone with respect to our partial order. To the best of our knowledge, both existence and monotonicity are novel in this setting. Moreover, monotonicity is here pivotal in establishing existence; the proof turns on passing from a sequence of monotone equilibria on discrete spaces to the limit of this sequence in a continuous space, which is feasible only because the space of monotone strategies is known to be compact in an almost-everywhere convergence metric if the partial

\footnotetext{
${ }^{3}$ In this section only, we restrict attention to simultaneous first-price auctions, as our primary purpose is illustration. We conjecture, however, that arguments similar to those in 5.1 could be used to establish existence of monotone equilibria in standard auctions more generally.
} 
order is sufficiently rich (Reny, 2011).

While we believe that this finding is of interest in its own right, this example also serves to highlight a subtle challenge arising in settings with more than one global bidder. Specifically, the interaction between strategic overbidding by global bidder $i$ and dependence across auctions of bids by $i$ 's global rivals leads to failure of a key technical property - better-reply security of Reny (1999) — needed to complete the existence proof. In Section 5.2, we therefore turn to an alternative solution concept, equilibrium with endogenous tiebreaking, building on the work of Jackson, Simon, Swinkels and Zame (2002, henceforth JSSZ). JSSZ define the communication extension $\mathcal{G}^{c}$ to a given game $\mathcal{G}$ as the game arising when, in addition to their actions under $\mathcal{G}$, players also submit cheap-talk indications of their types which the auctioneer may use (only) to resolve ties. A solution to $\mathcal{G}^{c}$ is a strategy profile for bidders plus a tiebreaking rule such that strategies are a Bayesian Nash equilibrium given the tiebreaking rule. Starting from a class of discontinuous games $\mathcal{G}$ which includes ours, JSSZ establish existence of solutions to $\mathcal{G}^{c}$ in which bidders play distributional strategies as defined by Milgrom and Weber (1985) and communication is truthful.

In the context of simultaneous auctions for complementary goods, we show that these general conclusions can be sharpened in at least three respects. First, rather than permitting bidders to communicate their full $\left(2^{L}-1\right) \times 1$-dimensional types, we allow bidder $i$ to submit (in addition to her bid vector $b_{i}$ ) only an $L \times 1$ vector of cheap-talk signals $s_{i}$; we refer to this as a signaling extension to distinguish it from the communication extension of JSSZ. Second, in any simultaneous standard auction, we show the existence of a solution to the signaling extension in which the auctioneer's tiebreaking rule can be characterized by a set of $L$ weakly monotone tiebreaking precedence functions $\left(\rho_{1}, \ldots, \rho_{l}\right)$, where the auctioneer randomizes object $l$ 
independently among the set of high bidders in auction $l$ with the highest tiebreaking precedence: i.e. among the set of bidders $i$ with $b_{i l}=\max _{j}\left\{b_{j l}\right\}$ and $\rho_{l}\left(s_{i l}\right)=$ $\max _{j}\left\{\rho_{l}\left(s_{j l}\right)\right\}$. This characterization of tiebreaking sharpens that in JSSZ, implying in particular existence of a solution where allocations and payments in auction $l$ depend only on bids and signals in auction $l$. Furthermore, whereas JSSZ consider only existence in distributional strategies, we obtain existence in pure strategies which are additionally monotone in a suitable partial order sense.

Section 6 contains several numerical examples of bidding behavior in simultaneous auctions for complementarities, which illustrate our main theoretical results. Proofs of all propositions and lemmas are collected in the Appendix.

Our analysis relates to an important phenomenon arising in simultaneous auctions known as the exposure problem. Exposure can occur when bidders try to win several objects without opportunities to express their preferences on combinations of the objects. In this situation, bidders might strategically underbid or overbid in an attempt to avoid winning undesirable bundles. As a result, the auctions can fail to construct efficient allocations, leading some bidders to incur ex-post losses. The exposure problem has been analyzed in various settings within the simultaneous auctions literature, although to the best of our knowledge the available results remain sparse. ${ }^{4}$ We do not analyse the exposure problem formally in the paper but we do highlight this problem in our examples.

Related literature The effect of complementarities in simultaneous auctions has been studied, among others and in very specific setups, by Bikhchandani (1999), by Rosenthal and Wang (1996) and Szentes and Rosenthal (2003) in simultaneous

\footnotetext{
${ }^{4}$ See Krishna and Rosenthal (1996), Rosenthal and Wang (1996), Milgrom (2000a), Goeree and Lien (2014), Meng and Gunay (2017), among others.
} 
first-price auctions, by Krishna and Rosenthal (1996) in simultaneous second-price auction, and by Brusco and Lopomo $(2002,2009)$ and Cramton (1997) in simultaneous ascending auctions.

Szentes and Rosenthal (2003) study the simultaneous first-price mechanism in a complete information setting with two identical players who compete via simultaneous first-price auctions for three identical objects. Their analysis highlights the challenges involved in study of the simultaneous first-price mechanism - even in relatively simple settings, equilibrium turns out to have subtle and surprising properties. Similar complexity arises in Krishna and Rosenthal (1996), who study a setting where many identical objects are auctioned via simultaneous second-price auctions to two types of bidders: global bidders, who bid in multiple auctions, and local bidders, who bid in one auction only. Global bidders' preferences are characterized by a onedimensional private type describing their valuation for each (identical) single object, with a deterministic, common knowledge synergy realized in the event of a multiple win. In contrast, we allow a much richer type space in which global bidders to have private valuations for each of the possible $\left(2^{L}-1\right) \times 1$ combinations of auctioned objects. We thereby take a significant step toward characterizing equilibrium in a broad class of simultaneous auction games.

Games of incomplete information with payoffs supermodular in actions have also been studied by, among others, Athey (2001), McAdams (2003) and Reny (2011). Vives (1990) established the existence of pure-strategy equilibria in Bayesian games when payoffs are supermodular and upper-semicontinuous in actions. This could provide an alternative path to establishing existence when the bid space is finite, but does not speak to monotone equilibria. Meanwhile, Van Zandt and Vives (2007) demonstrate existence of monotone pure strategy equilibria in games with supermod- 
ular utility assuming (among other conditions) that utility is continuous and exhibits increasing differences in own and rival actions. The latter condition does not hold even in finite bid spaces, and therefore cannot be applied in our setting. ${ }^{5}$

There is also a substantial literature analyzing properties of various combinatorial auction mechanisms. Notable studies in this literature include Cantillon and Pesendorfer (2006), Ausubel and Milgrom (2002), Ausubel and Cramton (2004), Cramton (2006), Krishna and Rosenthal (1996), Klemperer (2008, 2010), Milgrom (2000a, 2000b), to mention just a few. Detailed surveys of this literature are given in de Vreis and Vorha (2003) and Cramton et al. (2006). While these studies also consider settings where bidders have preferences over combinations, the theoretical problems generated by simultaneous bidding differ substantially from those encountered in true combinatorial mechanisms. ${ }^{6}$

\section{Simultaneous standard auctions with comple- mentarities}

Consider a setting in which $N$ risk-neutral bidders compete for $L$ prizes allocated via a class of mechanisms we call simultaneous standard auctions, defined as follows:

Definition 1 (Simultaneous standard auctions). We say that objects $l=1, \ldots, L$ are allocated via simultaneous standard auctions if the bidding mechanism is such that:

\footnotetext{
${ }^{5}$ As usual in auctions, once one moves from finite to continuous bid spaces, utility is no longer either continuous or semi-continuous in actions. Hence results based on these no longer apply.

${ }^{6}$ Though only tangentially related to our problem, there is also a growing literature on multi-unit discriminatory auctions of homogeneous objects. Reny (1999, 2011), Athey (2001), and McAdams (2006) address existence and properties of equilibrium in such auctions. Meanwhile, Hortacsu and Puller (2008), Hortacsu and McAdams (2010), and Hortacsu (2011) provide more empirical perspectives on multi-unit auctions.
} 
1. Bidders may bid for each object $l=1, \ldots, L$ individually, but may not submit combination or contingent bids;

2. Each object $l$ is allocated to a high bidder in auction l, with payments conditional on allocation determined solely by bids in auction $l$.

Note that while allocations are always to a high bidder, payment rules need not be the same across $l$. In what follows, we frame discussion in terms of a single seller, although this is inessential for our results.

For ease of exposition, in analyzing monotonicity and bidding we will initially assume that ties are broken randomly and independently across objects:

Assumption 1 (Independent tie-breaking). Ties are broken independently across auctions; tie-breaking does not depend on bidders' types.

We will maintain this assumption through Section 5.1, which demonstrates existence of monotone equilibria in continuous bid spaces with one global bidder. It will be dropped in Section 5.2, when we consider monotone equilibria with endogenous tiebreaking in continuous bid spaces with many global bidders.

Let an outcome from the perspective of bidder $i$ be an $L \times 1$ indicator vector $\omega$ with a 1 in the $l$ th place if object $l$ is allocated to bidder $i$ and a 0 in the $l$ th place otherwise. Similarly, let the outcome matrix $\Omega$ for bidder $i$ be the $\left(2^{L}-1\right) \times L$ matrix whose rows contain (transposes of) each possible outcome $\omega \neq 0$ : e.g. if $L=2$,

$$
\Omega^{T}=\left[\begin{array}{lll}
0 & 1 & 1 \\
1 & 0 & 1
\end{array}\right]
$$

In what follows, we use the squared Euclidean norm $\|\omega\|^{2}$ to denote the number of objects allocated to bidder $i$ in outcome $\omega$. 
Bidder preferences Let $Y_{i}^{\omega}$ denote the combinatorial valuation bidder $i$ assigns to outcome $\omega$. We normalize the outcome "win nothing" to zero, and assume that valuations are non-decreasing in the set of objects won:

Assumption 2 (Values Normalized and Non-decreasing). $Y_{i}^{0}=0$ and $Y_{i}^{\omega}$ is nondecreasing in the vector of objects won: $\omega^{\prime} \geq \omega$ implies $Y_{i}^{\omega^{\prime}} \geq Y_{i}^{\omega}$.

Let $Y_{i}$ be the $\left(2^{L}-1\right) \times 1$ vector describing the combinatorial valuations $i$ assigns to all possible winning outcomes (normalizing $Y_{i}^{0}=0$ as above), with elements of $Y_{i}$ corresponding to rows in $\Omega$. In what follows, we interpret $Y_{i}$ as bidder $i$ 's private type in the bidding game, known to bidder $i$ but unknown to rivals at the time of bidding. We further assume that private types $Y_{i}$ are i.i.d. across bidders:

Assumption 3 (Independent Private Values). Each bidder $i$ draws private type $Y_{i}$ from a continuous c.d.f. $F_{Y, i}$ with compact support $\mathcal{Y}_{i} \subset R^{2^{L}-1}$, with $F_{Y, i}$ common knowledge and types drawn independent across bidders: $Y_{i} \perp Y_{j}$ for all $i, j$.

As our focus is on monotone equilibria, we will further assume that objects are complements in the sense that combinatorial valuations are supermodular in the set of objects won:

Definition 2. We will say that bidders have supermodular valuations if for any outcomes $\omega_{1}, \omega_{2}$,

$$
Y_{i}^{\omega_{1} \wedge \omega_{2}}+Y_{i}^{\omega_{1} \vee \omega_{2}} \geq Y_{i}^{\omega_{1}}+Y_{i}^{\omega_{2}}
$$

where $\omega_{1} \wedge \omega_{2}$ denotes the meet of $\omega_{1}, \omega_{2}$ and $\omega_{1} \vee \omega_{2}$ denotes the join of $\omega_{1}, \omega_{2}$.

Supermodularity implies that winning a larger set of objects increases the marginal valuation $i$ assigns to any additional object. 
Actions and strategies Let $\mathcal{B}_{i l}$ be the set of feasible bids for bidder $i$ in auction l. For each bidder $i$, we assume that $\mathcal{B}_{i l}$ is a compact subset of $\mathbb{R}^{+}{ }^{7}$ The action space for bidder $i$ is the set of $L \times 1$ bid vectors $b_{i}=\left(b_{i 1}, \ldots, b_{i L}\right)^{T}$, with $b_{i} \in \mathcal{B}_{i}=$ $\times_{l} \mathcal{B}_{i l}$ and $\mathcal{B}_{i}$ a lattice in $\mathbb{R}^{L}$. As usual, a pure strategy for bidder $i$ is a function $\sigma_{i}: \mathcal{Y}_{i} \rightarrow \mathcal{B}_{i}$. Let $\sigma=\left(\sigma_{1}, \ldots, \sigma_{N}\right)$ denote a pure strategy profile for all bidders, and $\sigma_{-i}=\left(\sigma_{1}, \ldots, \sigma_{i-1}, \sigma_{i+1}, \ldots, \sigma_{N}\right)$ denote a strategy profile for all bidders except $i .^{8}$

Joint and marginal winning probabilities Let $P_{i}\left(b ; \sigma_{-i}\right)$ be the $\left(2^{L}-1\right) \times 1$ vector describing the probability distribution over outcomes arising when $i$ submits bid $b \in \mathcal{B}_{i l}$ against rival strategies $\sigma_{-i}$, with $P_{i}^{\omega}\left(b ; \sigma_{-i}\right)$ the element of $P_{i}\left(b ; \sigma_{-i}\right)$ describing the probability of outcome $\omega$. Similarly, let $\Gamma_{i}\left(b ; \sigma_{-i}\right)$ be the $L \times 1$ vector describing marginal win probabilities arising when $i$ submits bid vector $b \in \mathcal{B}_{i l}$ against rival strategy profile $\sigma_{-i}$, with $\Gamma_{i l}\left(b ; \sigma_{-i}\right)$ the marginal probability $i$ wins auction $l$. Observe that $\Gamma_{i}\left(b ; \sigma_{-i}\right)$ is related to $P_{i}\left(b ; \sigma_{-i}\right)$ by

$$
\Gamma_{i}\left(b ; \sigma_{-i}\right)=\Omega^{T} P_{i}\left(b ; \sigma_{-i}\right) .
$$

Under Assumption 1, $\Gamma_{i l}\left(b ; \sigma_{-i}\right)$ depends only on bid $b_{l}$. Furthermore, if ties occur with probability zero, $\Gamma_{i l}\left(b ; \sigma_{-i}\right)$ is the c.d.f. of the maximum rival bid in auction $l$.

Interim payoffs and expected payments Let $\pi_{i}\left(b_{i} ; y_{i}, \sigma_{-i}\right)$ denote the expected interim payoff of bidder $i$ with type $y_{i} \in \mathcal{Y}_{i}$ submitting bid vector $b_{i}$ against rival

\footnotetext{
${ }^{7}$ While we do not explicitly model reserve prices, these can easily be accommodated in our framework by introducing a dummy bidder whose action space is a singleton including only the relevant reserve prices.

${ }^{8}$ Note that although we do not discuss reserve prices explicitly, our framing here in fact implicitly accommodates arbitrary reserve prices. One could, for instance, simply include a dummy bidder whose bid space in each auction is a singleton equal to the relevant reserve. All results developed below would then immediately extend.
} 
strategies $\sigma_{-i}$. Maintaining Assumptions 1-3, we may write $\pi_{i}\left(b_{i} ; y_{i}, \sigma_{-i}\right)$ as follows:

$$
\pi_{i}\left(b_{i} ; y_{i}, \sigma_{-i}\right)=y_{i}^{T} P_{i}\left(b ; \sigma_{-i}\right)-\sum_{l=1}^{L} c_{i l}\left(b_{i l} ; \sigma_{-i}\right),
$$

where $c_{i l}\left(b_{i l} ; \sigma_{-i}\right)$ denotes $i$ 's expected mechanism-determined payment in auction $l$ as a function of $i$ 's bid $b_{i l}$ in auction $l$ given rival strategies $\sigma_{-i}$. For example, if auction $l$ is a first-price auction, then we would have

$$
c_{i l}\left(b_{i l} ; \sigma_{-i}\right)=b_{i l} \Gamma_{i l}\left(b_{i l} ; \sigma_{-i}\right) .
$$

Note that the additively separable form for payments follows jointly from our hypotheses of standard auctions and independent tiebreaking; the former implies that payments in auction $l$ depend only on allocations and bids in auction $l$, while the latter implies that allocations in auction $l$ depend only on bids in auction $l$.

\section{Monotone best responses in simultaneous stan- dard auctions with complementarities}

\subsection{Partial order on types}

A natural first question in analysis of simultaneous auctions is whether bidding strategies are monotone in any natural economic sense. Monotonicity is useful in analyzing both technical and empirical questions. Furthermore, insofar as we are focusing on a setting with complementarities between objects, it is natural to expect that "higher valuations" should in some sense translate into higher bids. In this section, we show that this is in fact the case: there is a partial order $\succeq$ on the space of types $\mathcal{Y}_{i}$ such 
that if $y_{i}^{\prime} \succeq y_{i}$, then $i$ has a best reply at type $y_{i}^{\prime}$ which is coordinatewise greater than any best reply at type $y_{i}$.

We first make precise the notion of marginal valuations in this combinatorial context:

Definition 3 (Marginal valuations). Let $\omega$ and $\omega^{\prime}$ be two outcomes such that $\omega^{\prime} \geq \omega$ in the coordinatewise sense. For bidder $i$, the marginal valuation of objects corresponding to allocation $\omega^{\prime}$ relative to those in allocation $\omega$ is defined as the difference

$$
Y_{i}^{\omega^{\prime}}-Y_{i}^{\omega}
$$

Recall that under Assumption 2 all marginal valuations are non-negative.

We define a partial order $\succeq$ on the space of types $\mathcal{Y}_{i}$ such that $y_{i}^{\prime} \succeq y_{i}$ implies that every marginal valuation is higher for type $y_{i}^{\prime}$ than for type $y_{i}$. Bearing in mind the combinatorial nature of marginal valuations, this leads to the following definition for the partial order $\succeq$ :

Definition 4 (Partial order). We will say that

$$
\tilde{y}_{i} \succeq y_{i}
$$

if and only if for any outcome $\omega$ and any object $l$ such that $\omega_{l}=0$ we have

$$
\tilde{y}_{i}^{\omega \vee e_{l}}-\tilde{y}_{i}^{\omega} \geq y_{i}^{\omega \vee e_{l}}-y_{i}^{\omega}
$$

Note that (by construction) the partial order $(P O)$ is more restrictive than the usual coordinatewise order on $\mathcal{Y}_{i}$. In particular, choosing $\omega=0$, we find that $\tilde{y}_{i} \succeq y_{i}$ implies $\tilde{y}_{i}^{e_{l}} \geq y_{i}^{e_{l}}$ for any object $l$, which in turn implies $\tilde{y}_{i}^{e_{l} \vee e_{m}} \geq y_{i}^{e_{l} \vee e_{m}}$ for any $l \neq m$ and so forth. Proceeding inductively in this way, we ultimately conclude that $\tilde{y}_{i} \succeq y_{i}$ implies $\tilde{y}_{i} \geq y_{i}$ coordinatewise.

Importantly, the coordinatewise partial order on the set of combinatorial valuations would not be sufficient to ensure coordinate-wise monotonicity of best re- 
sponses; indeed, even when objects are complements, a strict coordinatewise increase in $i$ 's type can lead to a strict coordinatewise decrease in all elements of $i$ 's bestresponse bid. Intuitively, this is because the usual coordinatewise order on $\mathcal{Y}_{i}$ imposes insufficient structure on the marginal value added by any additional object.

The failure of coordinatewise monotonicity in the context of a two-object simultaneous first price example is illustrated in Example 1.

Example 1. Consider two bidders competing for two objects via simultaneous firstprice auctions. Suppose that bidder 2's fixed strategy is to bid either in auction 1 (with probability $\frac{1}{2}$ ) or in auction 2 (with probability $\frac{1}{2}$ ), drawing bids from the uniform $U[0 ; 1]$ distribution in either case. Consider two types for bidder 1 :

$$
y_{1}^{\prime}=(0,0,0,2)^{T}, \quad y_{1}^{\prime \prime}=(0,1,1,5 / 2)^{T} .
$$

For $b_{1}, b_{2} \in[0,1]$, these types correspond to the following profit functions:

$$
\begin{aligned}
& \pi^{\prime}=-b_{1} \cdot\left(\frac{1}{2}-\frac{b_{2}}{2}\right)-b_{2} \cdot\left(\frac{1}{2}-\frac{b_{1}}{2}\right)+\left(2-b_{1}-b_{2}\right) \cdot \frac{b_{1}+b_{2}}{2} \\
& \pi^{\prime \prime}=-b_{1} \cdot\left(\frac{1}{2}-\frac{b_{2}}{2}\right)+\left(\frac{5}{2}-b_{2}\right) \cdot\left(\frac{1}{2}-\frac{b_{1}}{2}\right)+\left(\frac{5}{2}-b_{1}-b_{2}\right) \cdot \frac{b_{1}+b_{2}}{2}
\end{aligned}
$$

yielding best response bids $b^{\prime}=\left(\frac{1}{2}, \frac{1}{2}\right)^{T}$ and $b^{\prime \prime}=\left(\frac{1}{4}, \frac{1}{4}\right)^{T}$ respectively. Ignoring the first component, which corresponds to the case of winning no auctions, we see that $y_{1}^{\prime \prime}$ is strictly greater than $y_{1}^{\prime}$ in the coordinatewise sense.

Thus even when objects are complements in the (strong) sense of supermodular valuations, a strict coordinatewise increase in type (from $y_{1}^{\prime}$ to $y_{1}^{\prime \prime}$ ) can generate a strict coordinatewise decrease in $i$ 's best response bid (from $b^{\prime}$ to $b^{\prime \prime}$ ). As pointed out by Reny (2011) in a substantially different context (multi-unit auctions with riskaverse bidders), the fundamental problem is that the coordinatewise partial order on types imposes insufficient structure on marginal value added: for instance, when moving from $y_{1}^{\prime}$ to $y_{1}^{\prime \prime}$ in Example 1, the value added by object 2 (in events where $i$ is already winning object 1 ) falls from 2 to 1.5 even as the value $i$ assigns to winning 
objects 1 and 2 together increases from 2 to 2.5 .

Going back to the partial order $(P O)$, while the economic motivation for it is clear, we note that it also has a useful geometric interpretation. Intuitively, this interpretation arises from the observation that there is a positive cone generating our partial order. Specifically, define the set $\mathcal{Z}_{2^{L}-1}$ as follows:

$\mathcal{Z}_{2^{L}-1}=\left\{z \in \mathbb{R}^{2^{L}-1}:\right.$ taking $z^{0}=0, \quad \forall\left(\omega, l\right.$ such that $\left.\left.\omega_{l}=0\right) \quad z^{\omega \vee e_{l}}-z^{\omega} \geq 0\right\}$.

Then $\mathcal{Z}_{2^{L}-1}$ is a solid cone located in the first orthant of the $2^{L}$ - 1-dimensional space $\mathbb{R}^{2^{L}-1} \cdot{ }^{9}$ Furthermore, for any $y_{i} \in \mathbb{R}^{2^{L}-1}$, the set $y_{i}+\mathcal{Z}_{2^{L}-1}$, which amounts to the translation of the cone $\mathcal{Z}_{2^{L}-1}$ in $\mathbb{R}^{2^{L}-1}$ to vertex $y_{i}$, represents all the realizations of $i$ 's type in $\mathbb{R}^{2^{L}-1}$ that dominate $y_{i}$ in the sense of partial order $(P O)$. Similarly, the set $y_{i}-\mathcal{Z}_{2^{L}-1}$, which amounts to the rotation of $\mathcal{Z}_{2^{L}-1}$ and then its translation in $\mathbb{R}^{2^{L}-1}$ to vertex $y_{i}$, represents all the realizations of $i$ 's type in $\mathbb{R}^{2^{L}-1}$ that are dominated by $y_{i}$ under the partial order $(P O)$.

\subsection{Monotone best replies}

To conclude this section, we demonstrate that the additional structure imposed by the partial order $(P O)$ is in fact sufficient to restore an economically meaningful notion of monotonicity. Specifically, given any set of rival strategy profiles $\sigma_{-i}$, we show that if $\tilde{y}_{i} \succeq y_{i}$ in the sense of $(P O)$, then at least one element of $i$ 's best reply

\footnotetext{
${ }^{9}$ To remind the readers, a nonempty subset $Z$ of a vector space is said to be a cone if it satisfies the following three properties: (i) $Z+Z \subset Z$, (ii) $\alpha Z \subset Z$ for all $\alpha \geq 0$, (iii) $Z \cap(-Z)=\{0\}$. Any cone with a nonempty interior is a solid cone. In this case the interior of $Z^{2^{L}-1}$ is clearly nonempty in $\mathbb{R}^{2^{L}-1}$; e.g. for $L=2$ we have $\left(z^{e_{1}}, z^{e_{2}}, z^{e_{1} \vee e_{2}}\right)^{T}=(1,1,2)^{T} \in \mathcal{Z}_{3}^{o}$, for $L=3$ we have $\left(z^{e_{1}}, z^{e_{2}}, z^{e_{3}}, z^{e_{1} \vee e_{2}}, z^{e_{1} \vee e_{3}}, z^{e_{2} \vee e_{3}}, z^{e_{1} \vee e_{2} \vee e_{3}}\right)^{T}=(1,1,1,2,2,2,3)^{T} \in \mathcal{Z}_{7}^{o}$, and so forth for $L>3$. Hence $\mathcal{Z}_{2^{L}-1}$ is a solid cone in $\mathbb{R}^{2^{L}-1}$.
} 
bid correspondence at type $\tilde{y}_{i}$ is coordinatewise no smaller than every element of $i$ 's best reply bid correspondence at type $y_{i}$. We prove this proposition in three steps.

First, combining our hypothesis of simultaneous standard auctions with Assumptions 1-3 above, we show that supermodularity of valuations in the set of objects won implies supermodularity of interim payoffs $\pi_{i}\left(b_{i} ; y_{i}, \sigma_{-i}\right)$ as a function of $b_{i}$ :

Lemma 1. Suppose that Assumptions 1-3 hold. Fix a rival pure strategy profile $\sigma_{-i}$. Let $y_{i}$ be a realization of bidder $i$ 's type. If valuations are supermodular in the sense of Definition 2, then the interim payoff function

$$
\pi_{i}\left(b_{i} ; y_{i}, \sigma_{-i}\right)=y_{i}^{T} P\left(b_{i} ; \sigma_{-i}\right)-\sum_{l=1}^{L} c_{i l}\left(b_{i l} ; \sigma_{-i}\right)
$$

is supermodular in $b_{i}$.

Second, we establish that bidders' interim payoffs satisfy the following weak single crossing property in $y_{i}$ :

Lemma 2. Maintaining Assumptions 1-3, fix a pure strategy $\sigma_{-i}$ for the rival bidders. Suppose that types $\tilde{y}_{i}$ and $y_{i}$ are such that $\tilde{y}_{i} \succeq y_{i}$ in the sense of the partial order (PO), and suppose that $\tilde{b}_{i} \geq b_{i}$ in the coordinatewise sense. Then

$$
\pi_{i}\left(\tilde{b}_{i} ; y_{i}, \sigma_{-i}\right) \geq \pi_{i}\left(b_{i} ; y_{i}, \sigma_{-i}\right) \quad \Longrightarrow \quad \pi_{i}\left(\tilde{b}_{i} ; \tilde{y}_{i}, \sigma_{-i}\right) \geq \pi_{i}\left(b_{i} ; \tilde{y}_{i}, \sigma_{-i}\right) .
$$

Finally, we combine these results to establish the following weak monotonicity property on the set of $i$ 's best replies to $\sigma_{-i}$ :

Proposition 1. Maintaining Assumptions 1-3, suppose that valuations are supermodular in the sense of Definition 2. Fix a pure strategy profile $\sigma_{-i}$ for the rival bidders. Let $b_{i} \in \mathcal{B}_{i}$ be a best response to $\sigma_{-i}$ when bidder $i$ 's type is $y_{i}$, and $\tilde{b}_{i} \in \mathcal{B}_{i}$ be a best response to $\sigma_{-i}$ when $i$ 's type is $\tilde{y}_{i}$, where $\tilde{y}_{i}$ and $y_{i}$ are such that $\tilde{y}_{i} \succeq y_{i}$ in the sense of the partial order (PO). Then the bid vector $b_{i} \vee \tilde{b}_{i}$ is also a best response to $\sigma_{-i}$ when $i$ 's type is $\tilde{y}_{i}$. 
Proposition 1 does not, of course, guarantee existence of a best reply strategy $\sigma_{i}^{*}$ to $\sigma_{-i}$. It does, however, imply that if such a best reply exists, then there also exists a best reply strategy $\tilde{\sigma}_{i}^{*}$ which is monotone in that $y_{i}^{\prime} \succeq y_{i}$ in the sense of $(P O)$ implies $\tilde{\sigma}_{i}^{*}\left(y_{i}^{\prime}\right) \geq \tilde{\sigma}_{i}^{*}\left(y_{i}\right)$ in the usual coordinatewise order. This in turn provides a foundation for our analysis of monotone equilibrium below.

\section{Monotone equilibrium in finite bid spaces}

Building on the partial order $(P O)$, we next turn to consider monotone equilibrium in finite bid spaces. Specifically, suppose that $\mathcal{B}$ is a finite lattice. Under an additional support condition on $Y_{i}$ to be defined shortly, we show that there exists a Bayesian Nash equilibrium in pure strategies $\left(\sigma_{1}^{*}, \ldots, \sigma_{N}^{*}\right): \mathcal{Y} \rightarrow \mathcal{B}$ with the property that $y_{i}^{\prime} \succeq y_{i}$ in the sense of $(P O)$ implies $\sigma_{i}^{*}\left(y_{i}^{\prime}\right) \geq \sigma_{i}^{*}\left(y_{i}\right)$ in the usual coordinatewise order.

Toward this end, we require one additional assumption, which guarantees that the support of $Y_{i}$ is "sufficiently rich" to permit meaningful comparisons with respect to the partial order $(P O)$ :

Assumption 4. There is a countable subset $\mathcal{Y}_{i}^{\star}$ of $\mathcal{Y}_{i}$ such that every set in $F_{Y_{i}}$ sigma-algebra assigned positive probability by $F_{Y_{i}}$ contains two points between which (here "between" is understood in the partial order sense) lies a point in $\mathcal{Y}_{i}^{\star}$.

For this assumption to hold jointly with atomlessness of the distribution of $Y_{i}$, it is necessary that there exists a positive $F_{Y_{i}}$-measure of points in the support $\mathcal{Y}_{i}$ that can be compared to each other by means of the partial order $(P O)$. In particular, $(P O)$ should not reduce to the trivial partial order on $\mathcal{Y}_{i}$. Fortunately, Assumption 4 turns out to follow from natural regularity conditions on the distribution of $Y_{i}$ : 
Proposition 2. Suppose that $\mathcal{Y}_{i}$ is compact and has non-empty interior with respect to $\mathbb{R}^{2^{L}-1}$, and that the boundary of $\mathcal{Y}_{i}$ has zero Lebesgue measure in $\mathbb{R}^{2^{L}-1}$. Further suppose that the joint distribution of the $\left(2^{L}-1\right)$-dimensional vector $Y_{i}$ has density. Then Assumption 4 holds.

Conditions in Proposition 2 are closer to the assumptions on distributions of types imposed by McAdams (2003), who in addition requires the density to be bounded from above and also be bounded away from zero on $\mathcal{Y}_{i}{ }^{10}$

We now turn to this section's main result: under Assumptions 1-4, at least one Bayesian Nash equilibrium monotone in the sense of $(P O)$ exists on any finite bid lattice. In view of Proposition 1, the proof of this statement is relatively straightforward. Restricting bids to a finite lattice guarantees the continuity of the interim payoff function, which ensures that each player's interim best reply correspondence is non-empty. Hence by Proposition 1, we conclude that each player has a best reply which is monotone and join closed with respect to the partial order $(P O)$ on types and the usual coordinatewise order on bids. To guarantee existence of an equilibrium in monotone pure strategies, we therefore need only verify conditions G.1-G.6 of Reny (2011), after which Theorem 4.1 of Reny (2011) delivers the result. Of these, only condition G.3 is potentially problematic, leading to our Assumption 4 and sufficient

\footnotetext{
${ }^{10}$ Note that McAdams (2003) obtains results only for the coordinatewise partial order and, thus, those results are not directly applicable in our general framework where the partial order is different. However, for some forms of the utilities for bundles we could apply McAdams (2003) results directly (if we, of course, strengthen the requirement of Proposition 2 on the distribution of types to conform with McAdams (2003)). Consider, for instance, the case when all standalone valuations $Y^{e_{\ell}}$ are distributed on $[0,1]$ and the value for a bundle $\omega,\|\omega\|>1$ ( $\|\cdot\|$ denotes the Euclidean norm), has the form$$
Y^{\omega}=\sum_{\ell=1}^{L} Y^{\omega \wedge e_{\ell}}+\sum_{\omega^{\prime}: \omega^{\prime} \leq \omega, \omega^{\prime} \neq \omega,\left\|\omega^{\prime}\right\|>1} z_{\omega^{\prime}} \prod_{h: \omega \wedge e_{h}=e_{h}} Y^{e_{h}},
$$

where the $z$-variables indicate degrees of complementarities of the objects given in their subscripts, and all $z$-variables are between 0 and 1 . It is clear that in this case we can just impose the coordinatewise order on both the standalone valuations $Y^{e_{\ell}}$ and the $z$-variables to obtain our results on the monotonicity of best responses and further results on equilibria applying McAdams (2003) directly. Note that our main results in this paper are more general as they do not require a specification (3) or similar, and also impose less stringent conditions on the distributions of types.
} 
conditions provided in Proposition 2. We thereby conclude:

Proposition 3. Maintaining Assumptions 1-4, suppose that valuations are supermodular in the sense of Definition 2. for each bidder $i$ the bid space $\mathcal{B}_{i} \subset \mathbb{R}^{L}$ is a finite lattice. ${ }^{11}$ Then there is an equilibrium in pure strategies which are monotone with respect to the partial order (PO) on types and the coordinatewise partial order on bids: i.e. such that $y_{i}^{\prime} \succeq y_{i}$ in the sense of (PO) implies $b_{i}^{\prime} \geq b_{i}$ in the usual coordinatewise sense.

As in Reny (2011), Proposition 3 immediately extends to existence of symmetric monotone equilibria when bidders are symmetric:

Corollary 1. In addition to the hypotheses of Proposition 3, suppose that bidders are symmetric in the sense that $\mathcal{Y}_{i}=\mathcal{Y}_{j}, F_{i}=F_{j}, \mathcal{B}_{i}=\mathcal{B}_{j}$ for all bidders $i, j$, and the i.i.d. tie-breaking rule is used. Then there is an equilibrium in symmetric pure strategies monotone with respect to the partial order (PO) on types and the coordinatewise partial order on bids.

The proof of this corollary follows immediately from the proof of Proposition 3 given in the Appenidix, but invoking Theorem 4.5 rather than Theorem 4.1 of Reny (2011). We therefore do not provide a separate proof.

Note that the main contribution of Proposition 3 is not existence per se, ${ }^{12}$ but rather existence in strategies which are monotone with respect to a suitably defined partial order. Since our analysis in Section 5 pivots on monotonicity, this additional structure turns out to be essential.

\footnotetext{
${ }^{11}$ E.g., $\mathcal{B}_{i}=\underset{l=1}{\times} \mathcal{B}_{i l}$ where each $\mathcal{B}_{i l}$ consists of the finite number of points.

${ }^{12}$ Existence of equilibrium in pure strategies could be obtained by, for instance, applying results in Milgrom and Weber (1985).
} 


\section{Monotone equilibria in continuous bid spaces}

We now turn from finite to continuous bid spaces, applying Proposition 3 to establish two new results. We begin with a special case inspired by Krishna and Rosenthal (1996), in which a single global bidder bids for multiple objects against many local bidders who bid for single objects only. We demonstrate existence of a monotone pure strategy Bayesian Nash equilibrium in this context, building on limiting techniques in Reny (2011) to extend from discrete to continuous bid spaces. While instructive in its own right, this example also highlights a subtle technical challenge: with more than one global bidder, interaction between strategic overbidding by global bidders and strategic dependence of bids across auctions leads to failure of a key technical condition-better-reply security of Reny (1999) — needed to complete the extension proof. Returning to the full model, we therefore consider instead a more general solution concept inspired by the work of JSSZ: equilibrium with endogenous tiebreaking. We define this solution concept in detail in Section 5.2, and demonstrate that equilibria with endogenous tiebreaking exist for any number of global bidders.

\subsection{One global bidder and many local bidders}

First consider the following special case of our general model: suppose that one global bidder competes for $L$ objects against many local bidders, with each local bidder competing in exactly one auction. Let bidder 0 denote the global bidder and let $l_{1}, \ldots, l_{N}$ denote the auctions in which local bidders $1, \ldots, N$ are competing.

For simplicity, and for this section only, further suppose that each auction $l=$ $1, \ldots, L$ is a first-price auction. We conjecture that similar results could be shown for other standard auctions, but do not pursue this further here as our goal is illustration. 
We specialize our assumptions to this environment as follows:

Assumption 5. The global bidder is risk-neutral and draws private type $Y_{0}$ (which contains combinatorial valuations for all $\omega \neq 0$ ) from a continuous $\left(2^{L}-1\right)$-variate c.d.f. $\quad F_{0}$ with compact support $\mathcal{Y}_{0} \subset \mathbb{R}^{2^{L}-1}$. The global bidder's valuations are monotone $\left(Y^{\omega^{\prime}} \geq Y^{\omega}\right.$ for $\left.\omega^{\prime} \geq \omega\right)$ and supermodular in the sense of Definition 2.

Assumption 6. Each local bidder $i$ is risk-neutral and draws private type $Y_{i}$ for object $l_{i}$ from a univariate continuous c.d.f. $F_{i}$ with compact support $\mathcal{Y}_{i}$ equal to the support of $Y_{0}^{e_{l_{i}}}, i=1, \ldots, N$. Types are independently distributed across all bidders.

For each $l=1, \ldots, L$, let $\mathcal{B}_{l} \subset \mathbb{R}$ be a compact interval describing feasible bids in auction $l$. Then a pure a strategy for the global bidder 0 is a map $\sigma_{0}: \mathcal{Y}_{0} \rightarrow \mathcal{B}_{0}$ with $\mathcal{B}_{0} \equiv \times_{l=1}^{L} \mathcal{B}_{l}$, while a pure strategy for local bidder $i$ is a map $\sigma_{i}: \mathcal{Y}_{i} \rightarrow \mathcal{B}_{l_{i}}$.

It is straightforward to construct a sequence of finite lattices $\left\{\ddot{\mathcal{B}}_{0}^{k} ; \ddot{\mathcal{B}}_{1}^{k}, \ldots, \ddot{\mathcal{B}}_{L}^{k}\right\}_{k=1}^{\infty}$ such that as $k \rightarrow \infty$ each $\ddot{\mathcal{B}}_{j}^{k}$ becomes increasingly dense in $\mathcal{B}_{j}, j=0, \ldots, L$. By Proposition 3, for each $k$ the collection of such finite lattices will induce a monotone equilibrium strategy profile $\ddot{\sigma}^{k}$, where monotonicity of the strategy of the global bidder is understood in the sense of the partial order $(P O)$ on the type space and the coordinatewise order for bid vectors, and monotonicity of the strategies of the local bidders are understood in the usual univariate sense. By Lemma A.13 in Reny (2011), the space of strategies monotone with respect to our partial order is compact in the pointwise convergence topology, hence the sequence of strategies $\left\{\ddot{\sigma}^{k}\right\}_{k=1}^{\infty}$ will have a subsequence $\left\{\ddot{\sigma}^{k_{j}}\right\}_{j=1}^{\infty}$ which converges pointwise a.e. to a limit $\sigma^{*}$. This limit $\sigma^{*}$ is a monotone pure strategy profile by construction; we seek to show that it also defines an equilibrium on the continuous bid space $\mathcal{B} \equiv\left\{\mathcal{B}_{0} ; \mathcal{B}_{1}, \ldots, \mathcal{B}_{L}\right\}$.

To achieve this, we apply the concept of better-reply security introduced in Reny (1999), defined formally as follows: 
Definition 5 (Secure a payoff). Player $i$ can secure a payoff of $\alpha \in \mathbb{R}$ at strategy profile $\sigma \in \mathcal{S}$ if there exists $\bar{\sigma}_{i} \in \mathcal{S}_{i}$ such that $\Pi_{i}\left(\bar{\sigma}_{i} ; \sigma_{-i}^{\prime}\right) \geq \alpha$ for all $\sigma_{-i}^{\prime}$ in some open neighborhood of $\sigma_{-i}$.

Definition 6 (Better-Reply Secure). A game $G=\left(\mathcal{S}_{i}, \Pi_{i}\right)_{i=1}^{N}$ is better-reply secure if whenever $(\tilde{\sigma}, \tilde{\Pi})$ is in the closure of the graph of the vector payoff function $\Pi(\cdot)$ and $\tilde{\sigma}$ is not an equilibrium, some player $i$ can secure a payoff strictly above $\tilde{\Pi}_{i}$ at $\tilde{\sigma}$.

By Remark 3.1 in Reny (1999) (p. 1038), if a game is better-reply secure, then the limit of a convergent sequence of $\epsilon$-equilibria, as $\epsilon$ tends to zero, is a pure strategy equilibrium. To establish existence of an equilibrium in monotone pure strategies on the continuous space $\mathcal{B}$, it is therefore sufficient to demonstrate the following:

(i) There exists a sequence of finite lattices $\left\{\ddot{\mathcal{B}}_{0}^{k} ; \ddot{\mathcal{B}}_{1}^{k}, \ldots, \ddot{\mathcal{B}}_{L}^{k}\right\}_{k=1}^{\infty}$ such that if $\ddot{\sigma}^{k}$ is a monotone pure strategy equilibrium on $\left\{\ddot{\mathcal{B}}_{0}^{k} ; \ddot{\mathcal{B}}_{1}^{k}, \ldots, \ddot{\mathcal{B}}_{L}^{k}\right\}$ for each $k$, then $\left\{\ddot{\sigma}^{k}\right\}_{k=1}^{\infty}$ is a sequence of $\epsilon$-equilibria on $\mathcal{B}$ for which $\epsilon \rightarrow 0$;

(ii) The bidding game is better-reply secure when bids may be submitted on $\mathcal{B}$.

We now establish each of these in turn.

Lemma 3. Suppose that Assumptions 1, 4, 5, and 6 hold, and that each auction $l=1, \ldots, L$ is a first-price auction. Let $\left\{\ddot{\mathcal{B}}_{0}^{k} ; \ddot{\mathcal{B}}_{1}^{k}, \ldots, \ddot{\mathcal{B}}_{L}^{k}\right\}_{k=1}^{\infty}$ be any sequence of finite lattices such that:

1. $\ddot{\mathcal{B}}_{0}^{k} \subset \mathcal{B}_{0}, \ddot{\mathcal{B}}_{l}^{k} \subset \mathcal{B}_{l}$ for all $l=1, \ldots, L$, and

$$
\mathcal{H}\left(\ddot{\mathcal{B}}_{j}^{k}, \mathcal{B}_{j}\right) \rightarrow 0 \text { as } k \rightarrow \infty
$$

for $j=0, \ldots, N$, where $\mathcal{H}(\cdot, \cdot)$ stands for the Hausdorff distance.

2. For each $l=1, \ldots, L, \ddot{\mathcal{B}}_{l}^{k}$ is a subset of $\ddot{\mathcal{B}}_{0 l}^{k}$ such that $\min \ddot{\mathcal{B}}_{0 l}^{k}<\min \ddot{\mathcal{B}}_{l}^{k}$, $\max \ddot{\mathcal{B}}_{0 l}^{k}>\max \ddot{\mathcal{B}}_{l}^{k}$, and for any $b_{l}^{\prime}, b_{l}^{\prime \prime} \in \ddot{\mathcal{B}}_{l}^{k}$ there exists a point $b_{l}^{\prime \prime \prime} \in \ddot{\mathcal{B}}_{0 l}^{k}$ such that $b_{l}^{\prime}<b_{l}^{\prime \prime \prime}<b_{l}^{\prime \prime}$.

Let $\ddot{\sigma}^{k}$ be a monotone pure strategy equilibrium for bid space $\ddot{\mathcal{B}}^{k}$. Then for any sequence $\left\{\epsilon^{m}\right\}$ such that $\epsilon^{m}>0$ and $\epsilon^{m} \rightarrow 0$, there exists a subsequence $\left\{k_{m}\right\}_{m=1}^{\infty}$ of $k=1,2, \ldots$ such that strategy profile $\ddot{\sigma}^{k_{m}}$ is an $\epsilon^{m}$-equilibrium on the unrestricted $\mathcal{B}$. 
Note that the proof of Lemma 3 turns on choosing lattices $\left\{\ddot{\mathcal{B}}_{0}^{k} ; \ddot{\mathcal{B}}_{1}^{k}, \ldots, \ddot{\mathcal{B}}_{1}^{k}\right\}$ such that the bid lattice of the global bidder is always finer than the product of the bid lattices of the local bidders. This guarantees that at each point along the sequence of finite grids considered the global bidder can resolve ties in the direction she most prefers. This in turn allows us to use the global bidder's revealed preference on finite lattices to bound her potential gains from deviation in continuous bid space.

Lemma 4. Suppose that Assumptions 1, 4, 5, and 6 hold, and that each auction $l=$ $1, \ldots, L$ is a first-price auction. Then the simultaneous first-price auction game with one global and many local bidders is better-reply secure when considering monotone strategies played by the bidders.

While we relegate details to the Appendix, we emphasize that the proof of Lemma 4 is more complicated here than in standard single- or multi-unit auctions. In typical single- or multi-unit auctions, better-reply security follows almost automatically from the fact that bidders almost surely bid below their marginal valuations. One can therefore construct payoff-securing deviations by slightly increasing bids at any point involving potential ties. ${ }^{13}$ Here, in contrast, the global bidder may strategically overbid for a given object - i.e. submit a bid strictly above her marginal valuationin the hope of winning higher-order combinations. The proof of Lemma 4 therefore in fact turns on independence of rival bids faced by the global bidder. This allows us to assert that any increase in the marginal probability of winning object $l$ proportionally increases the probability of winning all combinations involving object $l$, and hence to conclude that the global bidder will always want to break relevant ties in her favor.

Finally, combining Lemmas 3 and 4, Remark 3.1 in Reny (1999), and Lemma A.13 of Reny (2011) as described above, we obtain this subsection's main result:

\footnotetext{
${ }^{13}$ see, e.g., Reny $(1999,2011)$ for examples of this argument.
} 
Proposition 4. Suppose that Assumptions 1, 4, 5, and 6 hold, and that each auction $l=1, \ldots, L$ is a first-price auction. In the simultaneous first-price auction game with one global bidder and many local bidders, a monotone pure strategy equilibrium exists on the compact convex bid space $\mathcal{B}$.

Now consider what may go wrong with more than one global bidder. Recall that, due to the possibility of strategic overbidding by the global bidder, our proof of Lemma 4 (better-reply security) turns crucially on independence of bids by local rivals across auctions. Unfortunately, however, if multiple global bidders are present, bids by global rivals may in principle exhibit arbitrary dependence across auctions through strategies $\sigma$. When combined with the possibility of strategic overbidding, this turns out to imply that with multiple global bidders, the simultaneous auction game need not be better-reply secure.

For example, consider a setting in which two global bidders compete for two objects. Imagine a sequence of strategies along which these bidders converge to a tie in auction 1, such that at each strategy profile in the sequence, any type of bidder 1 bidding "just above" the tie point in auction 1 also submits a bid for object 2 which wins auction 2 with certainty. Then any deviation by bidder 2 to a point "just above" the tie will produce a strict increase in the probability that bidder 2 wins auction 1 , without increasing the probability that bidder 2 wins objects 1 and 2 together. If bidder 2 also engages in strategic overbidding for object 1, this could in turn imply a strict decrease in bidder 2's expected payoff. Hence even if the limit profile (with ties) is not an equilibrium, bidder 2 may not be able to secure any payoff higher than she eventually achieves along the sequence.

We illustrate such a case formally in Example 4 in the Appendix. This example confirms that, with more than one global bidder, the simultaneous auction game need not satisfy either better-reply security as defined by Reny (1999) or several 
recent extensions due to Carmona (2009), McLennan, Monteiro and Tourky (2013), and Barelli and Meneghel (2013) among others. This in turn undermines any proof of existence paralleling Proposition 4 with more than one global bidder.

\subsection{Monotone equilibrium with endogenous tie-breaking}

Recall that the technical challenge in establishing better-reply security with many global bidders is not overbidding per se; rather, it is the fact that by slightly increasing her bid in auction $l$, bidder $i$ may win auction $l$ only against types of global rivals against which $i$ is likely to lose in other auctions. There may therefore exist a sequence of strategies $\left\{\sigma^{k}\right\}_{k=1}^{\infty}$, converging to a limit $\tilde{\sigma}$ involving ties, such that no type tying at the limit can secure a higher payoff along the sequence, but a positive measure of tying types wish to deviate at the limiting profile $\tilde{\sigma}$. The fundamental problem in such a case is that independent tiebreaking at the limiting profile $\tilde{\sigma}$ may lose information regarding the order in which near-ties are broken along the sequence - types tying in the limit could submit different bids in auction $l$ at every strategy profile $\sigma^{k}$ in the sequence. If this tie-breaking order could be preserved in the limit, then $\tilde{\sigma}$ would in fact represent a monotone pure strategy equilibrium.

Motivated by this observation, in analyzing simultaneous auctions with many global bidders, we focus on a solution concept which generalizes Bayesian Nash equilibrium along the lines proposed by Jackson, Simon, Swinkels and Zame (2002, henceforth JSSZ). In what follows, we refer to this solution concept as an equilibrium with endogenous tiebreaking in the signaling extension of the simultaneous bidding game. We define this solution concept formally as follows.

Let $\mathcal{G}$ be a simultaneous auction game satisfying Assumptions 2-4 on primitives above. In what follows, we no longer presume that ties are resolved independently 
across actions according to some pre-specified tiebreaking rule - i.e. we no longer maintain Assumption 1 above. Rather, from this point forward, we interpret $\mathcal{G}$ as a game of indeterminate outcomes in the language of JSSZ: that is, a game requiring only that each object $l$ is awarded to a high bidder in auction $l$, without specifying which high bidder will receive the object in the event of a tie.

We define the signaling extension $\mathcal{G}^{s}$ to the game of indeterminate outcomes $\mathcal{G}$ by augmenting each bidder's strategy space as follows. For each auction $l$, we allow bidder $i$ to submit, in addition to her bid vector $b_{i}$, a vector of cheap-talk signal $s_{i} \in[0,1]^{L}$ indicating her desired tiebreaking precedence in each auction $l$. These signals are irrelevant for allocations and payoffs except in case of ties, in which case the auctioneer may consider $\left(s_{1}, \ldots, s_{N}\right)$ in determining how to break ties.

A pure strategy for bidder $i$ in the signaling extension $\mathcal{G}^{s}$ is therefore a function $\sigma_{i} \times \tau_{i}: \mathcal{Y}_{i} \rightarrow \mathcal{B}_{i} \times \mathcal{S}_{i}$, where $\sigma_{i}: \mathcal{Y}_{i} \rightarrow \mathcal{B}_{i}$ denotes $i$ 's bidding strategy as above and $\tau_{i}: \mathcal{Y}_{i} \rightarrow \mathcal{S}_{i}$ denotes $i$ 's tiebreaking strategy in the signaling extension. ${ }^{14}$ As above, let $\mathcal{Y}=\times_{i} \mathcal{Y}_{i}, \mathcal{B}=\times_{i} \mathcal{B}_{i}$, and $\mathcal{S}=\times_{i} \mathcal{S}_{i}$ collect type, bid, and signal spaces across bidders respectively, with $\sigma=\left(\sigma_{1}, \ldots, \sigma_{N}\right)$ and $\tau=\left(\tau_{1}, \ldots, \tau_{N}\right)$ denoting profiles of bidding and tiebreaking strategies across bidders.

For each auction $l \in\{1, \ldots, L\}$, let $\Delta_{l}^{N}$ be the $N$-dimensional simplex describing all possible probabilities with which object $l$ could be allocated to each of the $N$ competing bidders. Let the correspondence $\Theta: \mathcal{B} \times \mathcal{S} \rightarrow \Delta_{1}^{N} \times \cdots \times \Delta_{L}^{N}$ be the set of admissible object allocation probabilities in the signaling extension $\mathcal{G}^{s}$ to $\mathcal{G}$ : that is, the set of rules such that object $l$ is allocated to bidder $i$ only if $b_{i l}=\max _{j} b_{j l}$. Following JSSZ, we define an allocation rule $\theta: \mathcal{B} \times \mathcal{S} \rightarrow \Delta_{1}^{N} \times \cdots \times \Delta_{L}^{N}$ as a selection

\footnotetext{
${ }^{14}$ Since we focus on pure strategies, writing bidder $i$ 's strategy as the cross-product of her bidding and tiebreaking strategies involves no loss of generality.
} 
from the correspondence $\Theta .{ }^{15}$

As in JSSZ, we define a pure strategy solution to the signaling extension $\mathcal{G}^{s}$ as an allocation rule $\theta^{*}$ plus a profile of pure bidding and tiebreaking strategies $\sigma^{*} \times \tau^{*}$ such that $\sigma^{*} \times \tau^{*}$ constitute a Bayesian Nash equilibrium given the allocation rule $\theta^{*}$. We refer to any such solution as an equilibrium with endogenous tiebreaking.

We are now in position to state this section's main result: under our assumptions on primitives, there exists an equilibrium with endogenous tiebreaking in which (i) bidders play monotone pure strategies, (ii) allocation of object $l$ depends only on bids and signals in auction $l$, and (iii) tiebreaking is weakly monotone in signals.

Proposition 5. Let $\mathcal{G}$ be an $L$-object $N$-bidder simultaneous auction game. Suppose that assumptions 2-4 hold, that $\mathcal{B}_{i} \subset \mathbb{R}^{L}$ is a compact lattice for each $i=1, \ldots, N$, and that valuations are supermodular in the sense of Definition 2. Then the signaling extension $\mathcal{G}^{s}$ to $\mathcal{G}$ admits a pure strategy solution $\theta^{*} ; \sigma^{*} \times \tau^{*}$ such that:

1. Both bidding strategies $\sigma^{*}$ and signaling strategies $\tau^{*}$ are monotone with respect to the partial order (PO) on types and the coordinatewise order on actions;

2. The auctioneer's allocation rule $\theta^{*}$ can be characterized by a $L \times 1$ vector of anonymous tiebreaking precedence rules $\left(\rho_{1}, \ldots, \rho_{L}\right)$ such that:

(a) For each $l=1, \ldots, L, \rho_{l}$ is a weakly monotone function from $[0,1]$ to $[0,1]$;

(b) Bidder $i=1, \ldots, N$ submitting signal $s_{i l} \in[0,1]$ is assigned tiebreaking precedence $\rho_{l}\left(s_{i l}\right) \in[0,1]$ in auction $l$;

(c) Each object $l=1, \ldots, L$ is allocated randomly and uniformly among high bidders in auction $l$ with the highest tiebreaking precedence.

While we defer the full proof of Proposition 5 to the Appenidix, the intuition is worth sketching briefly here. As noted above, the fundamental problem in passing from the sequence of finite equilibria $\left\{\sigma^{k}\right\}_{k=1}^{\infty}$ to the limit $\sigma^{*}$ is that the relative

\footnotetext{
${ }^{15}$ Note that this set of allocation rules defines allocation probabilities over auctions, not over combinations; since we ultimately seek a set of allocation rules which are separable across auctions, this turns out to be sufficient for our purposes.
} 
tiebreaking precedence along the sequence need not be preserved in the limit. But since this relative tiebreaking precedence is determined (along the sequence) by the $L \times 1$ vectors of bids submitted by each bidder, it must be encodable in (at most) an additional $L \times 1$ of signals for each bidder. It only remains to find an encoding such that any bidder types separated by a non-vanishing measure of bids along the sequence also submit strictly different signals in the limit; we accomplish this by linking $\tau_{l}^{*}$ to the measure of the maximum bid in auction $l$. This construction implies that (almost) no type of bidder $i$ can strictly gain from submitting any $\left(b_{i}, s_{i}\right)$ in the range of equilibrium strategies $\times_{j}\left(\sigma_{j}^{*} \times \tau_{j}^{*}\right)$; otherwise, bidder $i$ could also eventually gain along the sequence $\left\{\sigma^{k}\right\}_{k=1}^{\infty}$ by bidding like the relevant type $y_{j}$ of bidder $j$. The role of the weakly monotone tiebreaking precendence rule $\rho_{l}$ is simply to rule out profitable off-equilibrium deviations by mapping every signal $s \in[0,1]$ to the same tiebreaking precedence as some on-equilibrium signal $s_{i}$ in the range of $\times_{i} \tau_{i}^{*}$. Hence, under $\rho_{l}$, bidder $i$ 's set of deviations in auction $l$ is payoff-equivalent to $i$ 's set of on-equilibrium deviations in auction $l$. In view of the observations above, this guarantees that $\sigma^{*} \times \tau^{*}$ is an equilibrium profile under $\rho$.

As with Proposition 3 above, Proposition 5 extends immediately to existence of symmetric equilibria with endogenous tiebreaking when bidders are symmetric:

Corollary 2. In addition to the hypotheses of Proposition 5, suppose that bidders are symmetric in the sense that $\mathcal{Y}_{i}=\mathcal{Y}_{j}, F_{i}=F_{j}$, and $\mathcal{B}_{i}=\mathcal{B}_{j}$ for all bidders $i, j$. Then the signaling extension $\mathcal{G}^{s}$ to $\mathcal{G}$ admits a pure strategy solution $\theta^{*} ; \sigma^{*} \times \tau^{*}$ satisfying the conclusions of Proposition 5 plus the property that strategies are symmetric: i.e. that $\sigma_{i}^{*} \times \tau_{i}^{*}=\sigma_{j}^{*} \times \tau_{j}^{*}$ for all bidders $i, j$.

The proof of this fact follows immediately from the proof of Proposition 5, replacing the generic sequence $\left\{\sigma^{k}\right\}$ of finite equilibria derived from Proposition 5 with a sequence of symmetric equilibria derived from Corollary 1. 
Although obviously related to JSSZ's general result on existence of equilibria with endogenous tiebreaking, Proposition 5 and Corollary 2 strengthen JSSZ's conclusions in several important respects. First, whereas JSSZ guarantee existence of a solution to the communication extension $\tilde{\mathcal{G}}^{c}$ only in distributional strategies as defined by Milgrom and Weber (1985), we obtain existence of such a solution in pure strategies which are additionally monotone with respect to the partial order $(P O)$. Second, we provide much more structure on tiebreaking than is available using only results in JSSZ. For instance, Proposition 5 yields a solution in which auctions are truly both simultaneous and separable: i.e. in which allocation of object $l$ depends only on bids and signals in auction $l$. Similarly, weak monotonicity of tiebreaking rules under Proposition 5 implies that allocation probabilities are weakly monotone in types.

\section{$6 \quad$ Examples}

Lastly, we present several examples illustrating bidding strategies, equilibrium overbidding, and the exposure problem in simultaneous auctions for complementary goods. We first explore a very simple discrete-bid environment where the bidding set consists of only two points, so that equilibrium bidding strategies can be solved analytically. We then proceed to explore bidding and exposure within a much richer bidding space, where equilibrium behavior can be approximated though not solved exactly.

Throughout this section, we consider a setting with two objects and two ex-ante symmetric global bidders. Each bidder has standalone valuation $Y_{i}^{1,0}$ for object one and $Y_{i}^{0,1}$ for object two, with all standalone valuations independent random variables distributed uniformly on $[0,1]$. Meanwhile, the joint valuation each bidder assigns 
to having two objects is

$$
Y_{i}^{(1,1)}=Y_{i}^{e_{1}}+Y_{i}^{e_{2}}+k, \quad i=1,2,
$$

where $k \geq 0$ denotes the complementarity each bidder associates with winning both objects, which for simplicity we assume is constant.

We begin with Example 2, in which we restrict both bidders to bid on a twoelement discrete grid. We consider three different auction formats - first-price, secondprice and all-pay —and illustrate symmetric BNE in pure monotone strategies in each. In this example, a bidder may potentially incur a loss when submitting the high bid, and whether such a loss happens depends on bidder's type as well as what that bidder won. This gives rise to potential exposure problems, whose incidence we quantify. In first-price and all-pay auctions, we find that exposure happens with positive probability for moderate and high levels of complementarity, with both the probability of exposure and the maximal degree of exposure increasing in $k$. For the second-price auction, the discrete bid grid implies that exposure may happen even for $k=0$, although the degree of exposure is again increasing in $k$. Looking at the maximal degree of exposure, we find that the exposure problem is generally no more severe in the all-pay auction than in the first-price auction, and less severe in the first-price auction than in the second-price auction.

Example 2. Consider the two-auction, two-bidder environment described above. Suppose the bidders are only allowed to bid either $b_{1}=0$ or $b_{2}=\frac{1}{2}$ for each object, and ties for objects are broken independently with a fair lottery. We consider firstprice, second-price, and all-pay auctions, characterizing a BNE in symmetric pure monotone strategies for each. We illustrate how BNE changes when $k$ increases and discuss occurrences of exposure.

\section{First-price auction}


If $k=0$, so that there are no complementarities from winning two objects, then each bidder always bidding 0 for each object is a BNE.

For $k \in(0,1)$, the equilibrium bidding strategies are

$$
\beta_{i}\left(Y_{i}^{e_{1}}, Y_{i}^{e_{2}}\right)=\left\{\begin{array}{lll}
\left(\frac{1}{2}, \frac{1}{2}\right), & \text { if } & \left(Y_{i}^{e_{1}}, Y_{i}^{e_{2}}\right) \in A, \\
\left.\frac{1}{2}, 0\right), & \text { if } & \left(Y_{i}^{e_{1}}, Y_{i}^{e_{2}}\right) \in B \\
\left.0, \frac{1}{2}\right), & \text { if } & \left(Y_{i}^{e_{1}}, Y_{i}^{e_{2}}\right) \in C, \\
(0,0), & \text { if } & \left(Y_{i}^{e_{1}}, Y_{i}^{e_{2}}\right) \in D
\end{array}\right.
$$

where regions $\mathrm{A}, \mathrm{B}, \mathrm{C}$ and $\mathrm{D}$ are defined as follows:

$$
\begin{aligned}
A & =\left\{\left(y^{e_{1}}, y^{e_{2}}\right) \in[0,1]^{2}: y^{e_{1}}+y^{e_{2}} \geq 2 \gamma(k)-\frac{k}{2}, y^{e_{1}} \geq \gamma(k)-\frac{k}{2}, y^{e_{2}} \geq \gamma(k)-\frac{k}{2}\right\}, \\
C & =\left\{\left(y^{e_{1}}, y^{e_{2}}\right) \in[0,1]^{2}: y^{e_{1}}<\gamma(k)-\frac{k}{2}, y^{e_{2}} \geq \gamma(k)\right\}, \\
B & =\left\{\left(y^{e_{1}}, y^{e_{2}}\right) \in[0,1]^{2}: y^{e_{1}} \geq \gamma(k), y^{e_{2}}<\gamma(k)-\frac{k}{2}\right\}, \\
D & =[0,1]^{2} \backslash(A \cup B \cup C),
\end{aligned}
$$

and

$$
\gamma(k)=\frac{\frac{k^{3}}{8}+\frac{3 k^{2}}{8}-\frac{k}{2}+1}{\frac{k^{2}}{2}+\frac{k}{2}+1} . \quad k \in(0,1] .
$$

Figure 1 illustrates the BNE in symmetric monotone pure strategies when $k=0.2$ and $k=0.6$.

For $k \in(1,2]$, the equilibrium bidding strategies are

$$
\beta_{i}\left(Y_{i}^{e_{1}}, Y_{i}^{e_{2}}\right)= \begin{cases}\left(\frac{1}{2}, \frac{1}{2}\right), & \text { if } \quad\left(Y_{i}^{e_{1}}, Y_{i}^{e_{2}}\right) \in A_{1} \\ (0,0), & \text { if } \quad\left(Y_{i}^{e_{1}}, Y_{i}^{e_{2}}\right) \in D_{1}\end{cases}
$$

where regions $\mathrm{A}$ and $\mathrm{D}$ are described as follows:

$$
\begin{aligned}
& D_{1}=\left\{\left(y^{e_{1}}, y^{e_{2}}\right) \in[0,1]^{2}: y^{e_{1}}+y^{e_{2}} \leq \gamma(k)\right\} \\
& A_{1}=[0,1]^{2} \backslash D
\end{aligned}
$$

with

$$
\gamma_{1}(k)=\frac{2-k}{\sqrt{1+(2-k)(k-1)}+1} .
$$

When $k \geq 2$, in the equilibrium bidders always submit $b_{2}=\frac{1}{2}$ for each object.

For $k>0$ small enough, there no exposure for any of the bidders. However, 


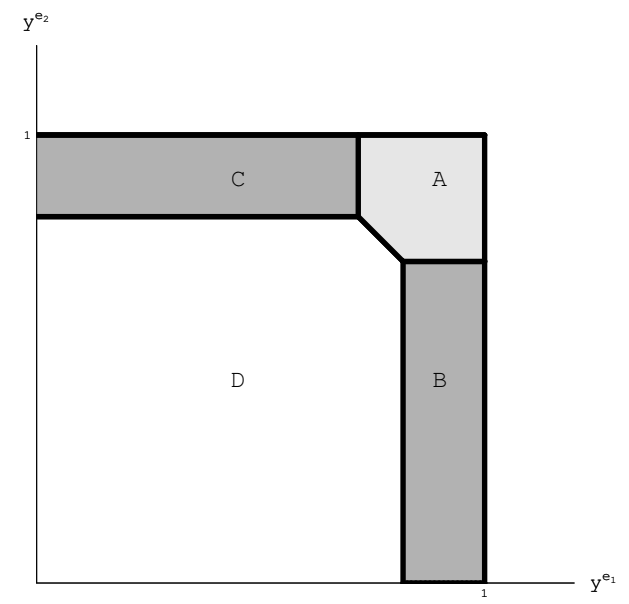

$k=0.2$

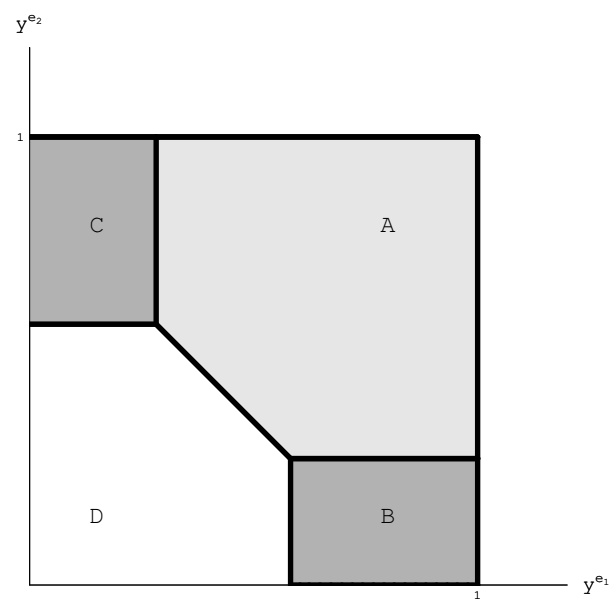

$k=0.6$

Figure 1: Illustration to Example 2 (first-price auction).

for $k \in\left(k_{f p, e x p}^{*}, 2\right]$ with $k_{f p, \exp }^{*} \approx 0.4171$ some bidders with valuations in region $A$ if $k \leq 1$ and valuations in region $A_{1}$ if $k \in(1,2]$ become exposed when winning one object only. For $k>2$ some bidders are also exposed when winning one object only. The maximum possible exposure (ex-post loss) is 0.5 .

\section{Second-price auction}

In a second-price auction, with $k=0$, the equilibrium bidding strategies are such that each bidder submits $b_{1}$ in auction $\ell$ if $y^{e_{\ell}}<\frac{1}{3}$ and submits $b_{2}$ otherwise.

When the complementarity becomes strictly positive, the equilibrium bidding strategies change. When $k \in\left(0, k_{s p}^{*}\right)$, where $k_{s p}^{*} \approx 0.8385$ (to be exact, $k_{s p}^{*}$ satisfies $\left.\frac{\left(k_{s p}^{*}\right)^{3}}{8}+\frac{\left(k_{s p}^{*}\right)^{2}}{8}+k_{s p}^{*}-1=0\right)$, then the monotone bidding strategies have the form (4)-(5) with

$$
\gamma(k)=\frac{\frac{k^{3}}{8}+\frac{5 k^{2}}{8}+\frac{k}{2}+1}{3+\frac{k^{2}}{2}+\frac{3 k}{2}} .
$$

The graphical illustration of these regions and equilibrium bidding behavior for $k=0.2$ and $k=0.6$ is given in Figure 2 .

For $k \in\left[k_{s p}^{*}, 2\right)$, the equilibrium bidding strategies are in the form (6)-(7) with

$$
\gamma_{1}(k)=\frac{2-k}{\sqrt{1+(2-k)(k+1)}+1} .
$$

For $k \geq 2$, in the equilibrium bidders always submit $b_{2}=\frac{1}{2}$ for each object. 


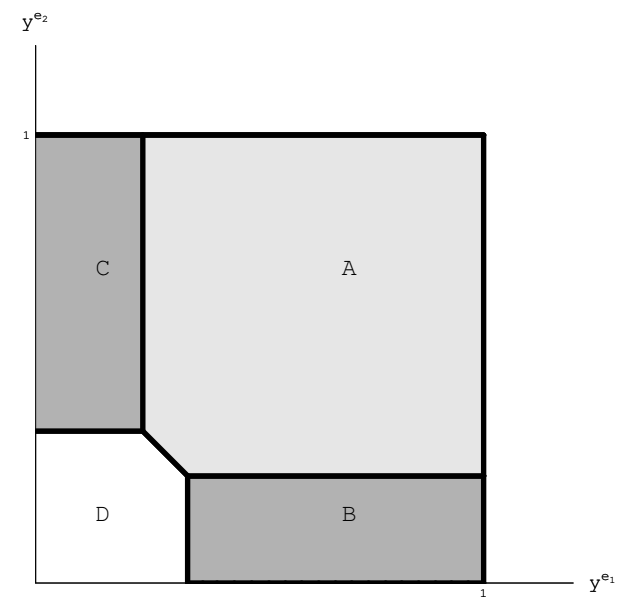

$k=0.2$

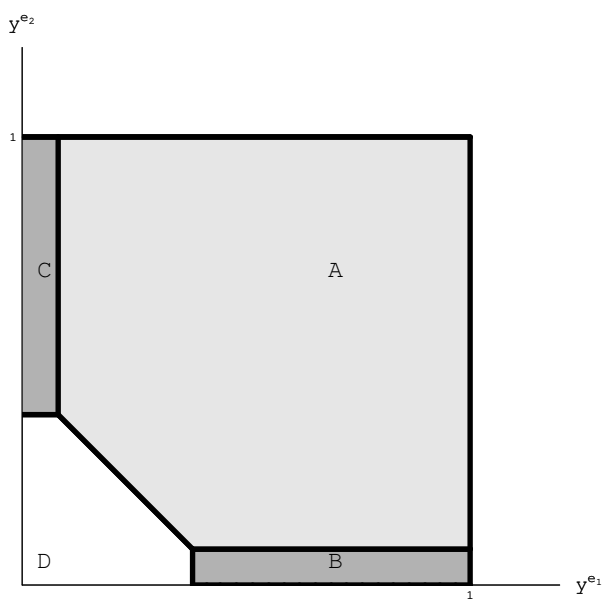

$k=0.6$

Figure 2: Illustration to Example 2 (second-price auction).

As we see, in this case bidders bid more aggressively than in the first-price auction.

Due to the second-price auction format, some bidders may be exposed even if $k=0$ when winning one object and having to pay $b_{2}$ for that object. The maximum possible degree of exposure in this situation is equal to $\frac{1}{3}$. When $k>0$, there are similar possible exposure situations when bidders with some values in in $A$ if $k \in\left(0, k_{s p}^{*}\right)$ or values in $A_{1}$ if $k \in\left(k_{s p}^{*}, 2\right)$ win one object only. However, in contrast to the first-price auction above, some bidders with values in these regions may be exposed even when winning both objects and having to pay $b_{2}$ for each object.

For $k \geq 2$, bidders always submit $b_{2}=\frac{1}{2}$ for each object. The maximum possible degree of exposure in this case is equal to 1 .

\section{All-pay auction}

In an all-pay auction, with $k=0$ the equilibrium bidding strategy is always submitting $b_{1}=0$ for each object.

When $k \in\left(0, k_{a p}^{*}\right)$, where $k_{a p}^{*} \approx 1.5418$ (to be exact, $k_{a p}^{*}$ satisfies $\frac{\left(k_{a p}^{*}\right)^{3}}{16}+\frac{k_{a p}^{*}}{2}-1=$ $0)$, the monotone bidding strategies have the form (4)-(5) with

$$
\gamma(k)=\frac{\frac{k^{3}}{16}+\frac{k^{2}}{4}+1}{\frac{k^{2}}{4}+\frac{k}{2}+1} .
$$

The graphical illustration of the equilibrium bidding strategies for $k=0.2$ and $k=0.6$ is given in Figure 3. 


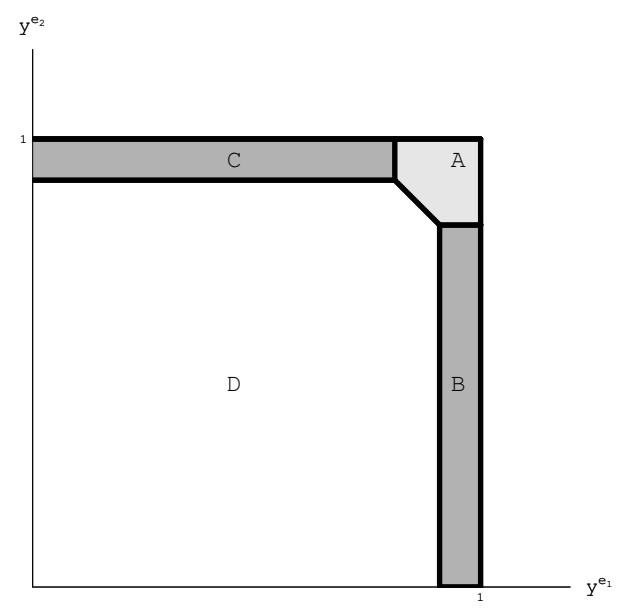

$k=0.2$

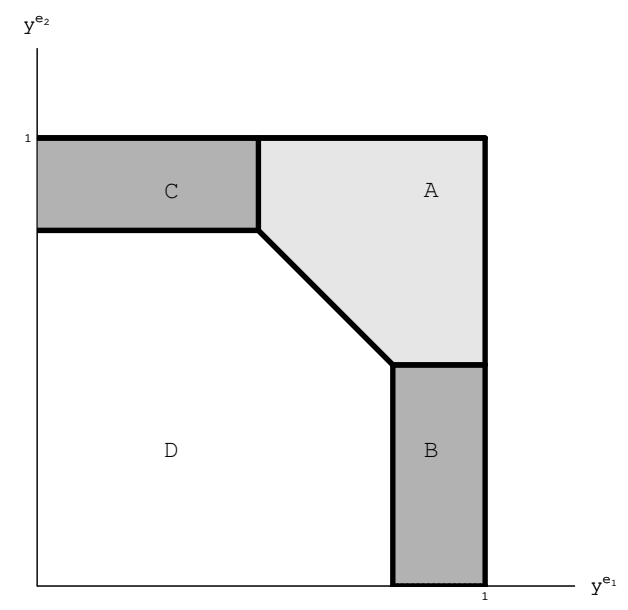

$k=0.6$

Figure 3: Illustration to Example 2 (all-pay auction).

For $k \in\left[k_{a p}^{*}, 4\right)$, the equilibrium bidding strategies are in the form (6)-(7) with

$$
\gamma_{1}(k)=\frac{4-k}{\sqrt{1+k(4-k)}+1} .
$$

For $k \geq 4$, bidders always submit $b_{2}=\frac{1}{2}$ for each object.

As we can see, bidders are more cautious in the all-pay auction than in the firstprice auction.

For $k>0$ small enough, there no exposure for any of the bidders.

For $k>k_{a p, e x p}^{*}$ with $k_{a p, e x p}^{*} \approx 0.5911$ some types in region $A$ if $k \leq k_{a p}^{*}$ and in in region $A_{1}$ if $k>k_{a p}^{*}$ are exposed when winning one object only. For $k \geq 4$, similarly some types will again be exposed when winning one object only. The maximum possible degree of exposure (ex-post loss) is 0.5 .

While Example 2 is simple enough that we can characterize equilibrium bidding strategies exactly, a two-element bid grid is obviously quite restrictive. To gain intuition regarding behavior in richer bidding spaces, we turn to Example 3, which numerically illustrates an approximate BNE in the same setting as Example 2, but now allowing a richer bidding space. For concreteness, we restrict attention to the 
simultaneous first-price auction format, illustrating strategies for the fixed complementarity level $k=0.3$. We then briefly discuss the effects of simultaneous bidding on exposure, revenue and efficiency in this simultaneous first-price setting.

Example 3. Consider the setting of Example 2, with two symmetric global bidders competing for two objects with fixed complementarity $k=0.3$. Suppose that objects are allocated via simultaneous first-price auctions, and that bidders can bid any non-negative price for each object.

Due to the constancy of the complementarity, the monotonicity of strategies $\beta_{\ell}$, $\ell=1,2$, in the partial order sense described in this paper is equivalent to monotonicity in $Y_{i}^{e_{1}}$ and in $Y_{i}^{e_{2}}$.

It is difficult to derive a closed form for the equilibrium bidding strategies even in this framework, so Figure 4 depicts approximations of these strategies obtained by the iteration of best responses on a dense discrete grid. Iteration is conducted until a pre-determined convergence criterion is satisfied. After such equilibrium best responses are obtained, we fit them on a continuous bidding by employing tensorproduct polynomials (in $Y_{i}^{e_{1}}, Y_{i}^{e_{2}}$ ) on $[1,2]^{2}$.

The monotonicity of strategies in each $Y^{e_{\ell}}, \ell=1,2$, can be clearly seen in Figure 4. For each fixed $Y_{i}^{e_{2}}$,the equilibrium bid for object 1 is strictly increasing in $Y_{i}^{e_{1}}$, and for each $Y_{i}^{e_{1}}$, increasing $Y_{i}^{e_{2}}$ also increases the equilibrium bidding strategy on object 1. Also, as expected, we have that for a given $Y^{e_{\ell}}$, each bidder submits a bid higher that in the case of additive valuations (zero complementarity).

Table 1 contains some numerical characteristics of economic aspects of this auction game. As we can see, the presence of a strictly positive complementarity in the valuation for both objects results in some exposure as well as revenue gains for the seller and lower expected profit for a bidder relative to the benchmark case of zero complementarity $\left(k=0\right.$ in $\left.Y_{i}^{(1,1)}=Y_{i}^{e_{1}}+Y_{i}^{e_{2}}+k\right)$. As is expected, auction simulteneity results in a positive probability of an inefficient allocation of an object.

\section{Conclusion}

Building on techniques in Athey (2001), McAdams (2003), Reny (2011) and JSSZ (2002), we establish the existence of pure strategy monotone equilibria in a class of standard simultaneous auction mechanisms for complementary goods. All the analysis is conducted under the assumption of supermodular preferences. In general 


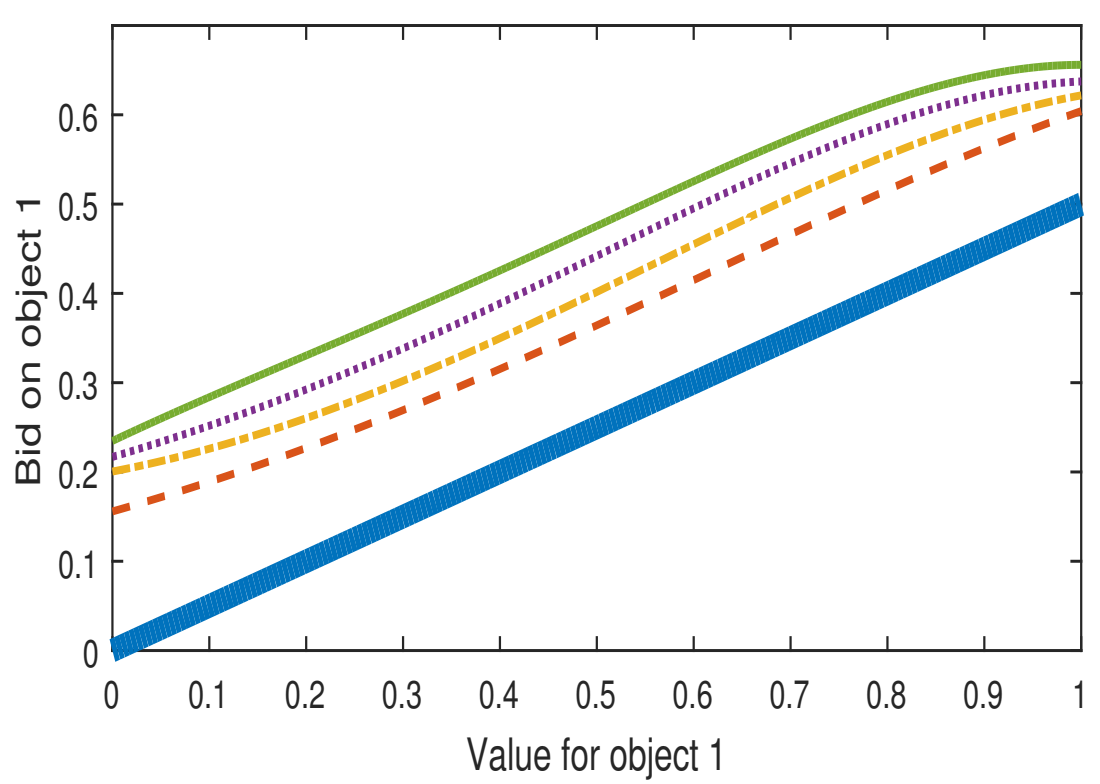

$k=0$
$---k=0.3$, value for object $2=0.25$
$k=0.3$, value for object $2=0.5$
$k=0.3$, value for object $2=0.75$
$k=0.3$, value for object $2=1$

Figure 4: Illustration of equilibrium bidding strategies in Example $3 . \quad k$ denotes the complementarity in $Y_{i}^{(1,1)}=Y_{i}^{e_{1}}+Y_{i}^{e_{2}}+k$.

bidders' valuations across different bundles of objects are not additive across individual objects in these bundles. Monotonicity on the space of types is understood in the partial order sense given by a cone described by a finite number of linear inequalities on increasing differences of bidders valuations between a larger and a smaller sets of objects, while monotonicity on the space of bids is understood in the coordinatewise order sense.

An alternative approach to existence could be the differential equations approach. 


\begin{tabular}{ll}
\hline \hline Expected seller's revenue from one auction & 0.4859 \\
Expected bidder's profit & 0.2777 \\
Prob of $i$ winning both objects & 0.3456 \\
Prob of $i$ winning only one object & 0.3079 \\
Prob of inefficiency when both objects are won by the same bidder & 0 \\
Prob of inefficiency in an auction when each bidder wins only one object & 0.0480 \\
Prob of exposure when both objects are won by the same bidder & 0 \\
Prob of exposure in an auction when each bidder wins only one object & 0.1241 \\
Average degree exposure in an auction (conditional on exposure) & 0.0777 \\
Average MLT in an auction & 0.1640 \\
Average MLT in an auction when winning only that auction & 0.1215 \\
Average total MLT when winning both auctions & 0.3655 \\
\hline
\end{tabular}

Table 1: Numerical results derived from simulation based on the bid strategies described in Example 3. MLT stands for money left on the table.

Because bidders' preferences are over many bundles of objects, the existence prob-

lem would be characterized by a system of partial differential equations with some boundary conditions. Issues of existence of solutions of systems of partial differential equations are infamously much more difficult than those in the systems of ordinary differential equations and, therefore, the approach did not seem realistic to us.

\section{References}

ATHEY, S. (2001), "Single Crossing Properties and the Existence of Pure Strategy Equilibria in Games of Incomplete Information", Econometrica, 69, 861-889.

ATHEY, S., LEVIN, J., and SEIRA, E. (2011), "Comparing Open and Sealed Bid Auctions: Evidence from Timber Auctions", The Quarterly Journal of Economics, 126, 207-257.

AUSUBEL, L.M., and CRAMTON, P. (2004), "Auctioning Many Divisible Goods", 
Journal of the European Economic Association, 2, 480-493, April-May.

AUSUBEL, L.M., and MILGROM, P. (2002), "Ascending auctions with package bidding," Frontiers of theoretical economics, 1(1), pp. 1-42.

BAGH, A., and JOFRE, A. (2006), "Reciprocal Upper Semicontinuity and Better Reply Secure Games: A Comment", Econometrica, 74(6), 1715-1721.

BAJARI, P., and FOX, J. (2013), "Measuring the Efficiency of an FCC Spectrum Auction", American Economic Journal: Microeconomics, 5(1), 100-146.

BARELI, P., and MENEGHEL, I. (2013), "On the Equilibrium Existence in Discontinuous Games", Econometrica, 81(2), 813-824.

BIKHCHANDANI, S. (1999), "Auctions of Heterogeneous Objects", Games and Economic Behavior, 26(2), 193-220.

BRUSCO S., and LOPOMO G. (2002), "Collusion via signaling in Simultaneous Ascending Bid Auctions with Heterogeneous Objects, with and without Complementarities", Review of Economic Studies, 69, 407-436.

BRUSCO S., and LOPOMO G. (2009), "Simultaneous Ascending Auctions with Complementarities and Known Budget Constraints", Economics Theory, 38, 105124.

CANTILlON, E. and PESENDORFER, M. (2006), "Combination Bidding in MultiUnit Auctions", (Mimeo).

CARMONA, G. (209), "An existence result for discontinuous games", Journal of Economic Theory, 144, 1333-1340.

COHN, D.L. (1980), Measure Theory (Boston, Birkhauser). 
CRAMTON, P. (1997), "The FCC Spectrum Auctions: An early Assessment", Journal of Economics and Management Strategy, 6, 647-675.

CRAMTON, P. (2006), "Simultaneous Ascending Auction", Ch. 4 in Cramton, P., Shoham, Y., and Steinberg, R. (eds) Combinatorial Auctions (Cambridge, MA: The MIT Press, 2006), 99-114.

CRAMton, P., SHOHAM, Y., and STEINBERG, R. (eds) (2006), Combinatorial Auctions (Cambridge, MA: MIT Press).

DE VREIS, S., and VORHA, R.V. (2003), "Combinatorial Auctions: A Survey", INFORMS Journal of Computing, 13(3), 284-309.

GENTRY, M., T. KOMAROVA, T., and P. SCHIRALDI (2015), "Simultaneous First-Price Auctions with Preferences over Combinations: Identification, Estimation and Application", Working Paper. Available at SSRN: http://ssrn.com/abstract=2514995.

GOEREE, J. K. and LIEN, Y., 2014. "An equilibrium analysis of the simultaneous ascending auction," Journal of Economic Theory, 153(C), 506-533.

GROEGER, J.R. (2012), "A Study of Participation in Dynamic Auctions", (Forthcoming at International Economic Review).

HENDRICKS, K., PINKSE, J., and PORTER, R. H. (2003), "Empirical Implications of Equilibrium Bidding in First-Price, Symmetric, Common Value Auctions", Review of Economic Studies, 70, 115-145.

HENDRICKS, K., and PORTER, R. H. (1988), "An Empirical Study of an Auction with Asymmetric Information", American Economic Review, 78(5), 865-883.

HORTACSU, A. (2011), "Recent Progress in the Empirical Analysis of Multi-Unit 
Auctions", International Journal of Industrial Organization, 29(3), 345-349.

HORTACSU, A. and McADAMS (2010), "Mechanism Choice and Strategic Bidding in Divisible Good Auctions: An Empirical Analysis of the Turkish Treasury Auction Market", Journal of Political Economy, 188(5), 833-865.

HORTACSU, A., and PULLER, S. L. (2008), "Understanding Strategic Bidding in Multi-Unit Auctions: A Case Study of the Texas Electricity Spot Market", RAND Journal of Economics, 39(1), 86-114.

JACKSON, M. O., SIMON, L. K., SWINKELS, J. M., and ZAME, W. R. (2002), "Communication and Equilibrium in Discontinuous Games of Incomplete Information", Econometrica, 70(5), 1711-1740.

KAWAI, K. (2011), "Auction Design and the Incentives to Invest: Evidence from Procurement Auctions", (Working Paper, New York University).

KLEMPERER, P. (2008), "A New Auction for Substitutes: Central Bank Liquidity Auctions, the U.S. TARP, and Variable Product-Mix Auctions. Bayesian Games and the Smoothness Framework", (Working Paper, Oxford University).

KLEMPERER, P. (2010), "The Product-Mix Auction: A new auction design for differentiated goods", Journal of the European Economic Association, 8, 526-536.

KRASNOKUTSKAYA, E. (2011), "Identification and Estimation in Procurement Auctions under Unobserved Auction Heterogeneity", Review of Economic Studies, 78(1), 293-327.

KRISHNA, V., and ROSENTHAL, R.W. (1996), "Simultaneous Auctions with Synergies", Games and Economic Behavior, 17, 1-31. 
LUNANDER, A., and LUNDBERG, S. (2013), "Bids and Costs in Combinatorial and Noncombinatorial Procurement Auctions - Evidence from Procurement of Public Cleaning Contracts", Contemporary Economic Policy, 31(4), 733-745.

McADAMS, D. (2003), "Isotone Equilibrium in Games of Incomplete Information", Econometrica, 71, 1191-1214.

McADAMS, D. (2006), "Monotone Equilibrium in Multi-Unit Auctions", Review of Economic Studies, 73, 1039-1056.

McLENNAN, M., MONTEIRO, P. K., and TOURKY, R. (2011), "Games with Discontinuous Payoffs: A strengthening of Reny's Existence Theorem", Econometrica, 79(5), 1643-1664.

MENG, X. and GUNAY, H. (2017). "Exposure Problem in Multi-unit Auctions", International Journal of Industrial Organization, 52, 165-187.

MILGROM, P. (2000a), "Putting Auction Theory to Work: The Simultaneous Ascending Auction", Journal of Political Economy, 108(2), 245-272.

MILGROM, P. (2000b), "Putting Auction Theory to Work: Ascending Auctions with Package Bidding", (Unpublished Working Paper).

MILGROM, P., and WEBER, R. (1985), "Distributional Strategies for Games with Incomplete Information", Mathematics of Operations Research, 10(4), 619-632.

RENY, P. J. (1999), "On the Existence of Pure and Mixed Strategy Nash Equilibria in Discontinuous Games", Econometrica, 67, 1029-1056.

RENY, P. J. (2011), "On the Existence of Monotone Pure Strategy Equilibria in Bayesian Games", Econometrica, 79, 499-553. 
ROSENTHAL, R.W., and WANG, R. (1996), "Simultaneous Auctions with Synergies and Common Values", Games and Economic Behavior, 17, 32-55.

SOMAINI, P. (2011), "Competition and Interdependent Costs in Highway Procurement", (Working Paper, Stanford University).

SZENTES, B., and ROSENTHAL, R.W. (2003), "Three-object Two-bidder Simultaneous Auctions: Chopsticks and Tetrahedra", Games and Economic Behavior, 44, 114-133.

VAN ZANDT, T., and VIVES, X. (1990), "Monotone equilibria in Bayesian games of strategic complementarities", Journal of Economic Theory, 134, 339-360.

VIVES, X. (1990), "Nash equilibrium with strategic complementarities", Journal of Mathematical Economics, 19, 305-321.

\section{Appendix: Proofs and counterexamples}

\section{Proof of Lemma 1}

Payments $\sum_{l=1}^{L} c_{i l}\left(b_{i l} ; \sigma_{-i}\right)$ are additively separable across auctions, hence modular in $b_{i}=$ $\left(b_{i l}, \ldots, b_{i l}\right)$ by construction. To establish supermodularity of interim payoffs

$$
\pi_{i}\left(b_{i} ; y_{i}, \sigma_{-i}\right)=y_{i}^{T} P\left(b_{i} ; \sigma_{i-}\right)-\sum_{l=1}^{L} c_{i l}\left(b_{i l} ; \sigma_{-i}\right)
$$

in $b_{i}$, it is therefore sufficient to establish that expected valuations $y_{i}^{T} P\left(b_{i} ; \sigma_{i-}\right)$ are supermodular in $b_{i}$ for any $y_{i} \in \mathcal{Y}_{i}$.

Toward this end, for any realization $y_{-i}$ of the rivals' types, let the $\left(\sum N_{l}-l\right) \times 1$ vector $B_{-i}=\sigma_{-i}\left(Y_{-i}\right)$ be the vector of all rival bids across all auctions. With slight abuse of notation, let $y_{i}\left(b ; B_{-i}\right)$ denote player $i$ 's expected valuation given type $y_{i}$, own bid vector $b$ and the complete rival bid vector $B_{-i}$ :

$$
y_{i}\left(b ; B_{-i}\right)=E_{\omega}\left[y_{i}^{\omega} \mid y_{i}, b, B_{-i}\right]
$$

where the expectation is taken over ties. Note that, in the absence of ties, bidder $i$ will 
win each auction in which his bid is the highest, $\omega=\omega\left(b, B_{-i}\right)$ will be deterministic, and $y_{i}\left(b ; B_{-i}\right)=y_{i}^{\omega\left(b, B_{-i}\right)}$.

The function $y_{i}^{T} P_{i}\left(b, \sigma_{-i}\right)$ can be written as the expectation of $y_{i}\left(b ; B_{-i}\right)$ with respect to the distribution of the rival bids:

$$
y_{i}^{T} P_{i}\left(b, \sigma_{-i}\right)=\int y_{i}\left(b ; \sigma_{-i}\left(Y_{-i}\right)\right) F_{-i}\left(d Y_{-i}\right)
$$

Let $b^{\prime}, b^{\prime \prime} \in \mathcal{B}_{i}$. We want to show that

$$
y_{i}^{T} P_{i}\left(b^{\prime \prime} \vee b^{\prime} ; \sigma_{-i}\right)+y_{i}^{T} P_{i}\left(b^{\prime \prime} \wedge b^{\prime} ; \sigma_{-i}\right) \geq y_{i}^{T} P_{i}\left(b^{\prime \prime} ; \sigma_{-i}\right)+y_{i}^{T} P_{i}\left(b^{\prime} ; \sigma_{-i}\right),
$$

or equivalently, that

$\int\left[y_{i}\left(b^{\prime \prime} \vee b^{\prime} ; \sigma_{-i}\left(Y_{-i}\right)\right)+y_{i}\left(b^{\prime \prime} \wedge b^{\prime} ; \sigma_{-i}\left(Y_{-i}\right)\right)-y_{i}\left(b^{\prime \prime} ; \sigma_{-i}\left(Y_{-i}\right)\right)-y_{i}\left(b^{\prime} ; \sigma_{-i}\left(Y_{-i}\right)\right)\right] F_{Y_{-i}}\left(d Y_{-i}\right) \geq 0$

For a specific realization $y_{-i}$ of rivals' types, if there are no ties for $\left(b^{\prime \prime} ; \sigma_{-i}\left(y_{-i}\right)\right)$ and $\left(b^{\prime} ; \sigma_{-i}\left(y_{-i}\right)\right)$, then

$$
\begin{aligned}
& \omega\left(b^{\prime \prime} \wedge b^{\prime} ; \sigma_{-i}\left(y_{-i}\right)\right)=\omega\left(b^{\prime} ; \sigma_{-i}\left(y_{-i}\right)\right) \wedge \omega\left(b^{\prime \prime} ; \sigma_{-i}\left(y_{-i}\right)\right) \\
& \omega\left(b^{\prime \prime} \vee b^{\prime} ; \sigma_{-i}\left(y_{-i}\right)\right)=\omega\left(b^{\prime} ; \sigma_{-i}\left(y_{-i}\right)\right) \vee \omega\left(b^{\prime \prime} ; \sigma_{-i}\left(y_{-i}\right)\right)
\end{aligned}
$$

and

$$
\begin{aligned}
& y_{i}\left(b^{\prime \prime} \vee b^{\prime} ; \sigma_{-i}\left(y_{-i}\right)\right)+y_{i}\left(b^{\prime \prime} \wedge b^{\prime} ; \sigma_{-i}\left(y_{-i}\right)\right)-y_{i}\left(b^{\prime \prime} ; \sigma_{-i}\left(y_{-i}\right)\right)-y_{i}\left(b^{\prime} ; \sigma_{-i}\left(y_{-i}\right)\right. \\
= & y_{i}^{\omega\left(b^{\prime \prime} \vee b^{\prime} ; \sigma_{-i}\left(y_{-i}\right)\right)}+y_{i}^{\omega\left(b^{\prime \prime} \wedge b^{\prime} ; \sigma_{-i}\left(y_{-i}\right)\right)}-y_{i}^{\omega\left(b^{\prime} ; \sigma_{-i}\left(y_{-i}\right)\right)}-y_{i}^{\omega\left(b^{\prime \prime} ; \sigma_{-i}\left(y_{-i}\right)\right)} \\
= & y_{i}^{\omega\left(b^{\prime} ; \sigma_{-i}\left(y_{-i}\right)\right) \vee \omega\left(b^{\prime \prime} ; \sigma_{-i}\left(y_{-i}\right)\right)}+y_{i}^{\omega\left(b^{\prime} ; \sigma_{-i}\left(y_{-i}\right)\right) \wedge \omega\left(b^{\prime \prime} ; \sigma_{-i}\left(y_{-i}\right)\right)}-y_{i}^{\omega\left(b^{\prime} ; \sigma_{-i}\left(y_{-i}\right)\right)}-y_{i}^{\omega\left(b^{\prime \prime} ; \sigma_{-i}\left(y_{-i}\right)\right)} \geq 0 .
\end{aligned}
$$

Alternatively, if there are ties at either $\left(b^{\prime \prime} ; \sigma_{-i}\left(y_{-i}\right)\right)$ or $\left(b^{\prime} ; \sigma_{-i}\left(y_{-i}\right)\right)$, then some of the outcome vectors $\omega\left(b^{\prime \prime} \wedge b^{\prime} ; \sigma_{-i}\left(y_{-i}\right)\right), \omega\left(b^{\prime \prime} \vee b^{\prime} ; \sigma_{-i}\left(y_{-i}\right)\right), \omega\left(b^{\prime} ; \sigma_{-i}\left(y_{-i}\right)\right), \omega\left(b^{\prime \prime} ; \sigma_{-i}\left(y_{-i}\right)\right)$ will be stochastic. Let $\lambda_{2}\left(b^{\prime} ; b^{\prime \prime} ; \sigma_{-i}\left(y_{-i}\right)\right)$ denote the joint distribution of $\omega\left(b^{\prime} ; \sigma_{-i}\left(y_{-i}\right)\right)$ and $\omega\left(b^{\prime \prime} ; \sigma_{-i}\left(y_{-i}\right)\right)$. Note that the distribution of $\omega\left(b^{\prime \prime} \vee b^{\prime} ; \sigma_{-i}\left(y_{-i}\right)\right)$ coincides with the distribution of $\omega\left(b^{\prime} ; \sigma_{-i}\left(y_{-i}\right)\right) \vee \omega\left(b^{\prime \prime} ; \sigma_{-i}\left(y_{-i}\right)\right)$, and the distribution of $\omega\left(b^{\prime \prime} \wedge b^{\prime} ; \sigma_{-i}\left(y_{-i}\right)\right)$ coincides with the distribution of $\omega\left(b^{\prime} ; \sigma_{-i}\left(y_{-i}\right)\right) \wedge \omega\left(b^{\prime \prime} ; \sigma_{-i}\left(y_{-i}\right)\right)$. Therefore,

$$
\begin{aligned}
& y_{i}\left(b^{\prime \prime} \vee b^{\prime} ; \sigma_{-i}\left(y_{-i}\right)\right)=E_{\left(\omega_{1}, \omega_{2}\right) \sim \lambda_{2}\left(b^{\prime} ; b^{\prime \prime} ; \sigma_{-i}\left(y_{-i}\right)\right)}\left[y_{i}^{\omega_{1} \vee \omega_{2}} \mid y_{i}, b, \sigma_{-i}\left(y_{-i}\right)\right], \\
& y_{i}\left(b^{\prime \prime} \wedge b^{\prime} ; \sigma_{-i}\left(y_{-i}\right)\right)=E_{\left(\omega_{1}, \omega_{2}\right) \sim \lambda_{2}\left(b^{\prime} ; b^{\prime \prime} ; \sigma_{-i}\left(y_{-i}\right)\right)}\left[y_{i}^{\omega_{1} \wedge \omega_{2}} \mid y_{i}, b, \sigma_{-i}\left(y_{-i}\right)\right] .
\end{aligned}
$$

Since we can write

$$
\begin{aligned}
& y_{i}\left(b^{\prime} ; \sigma_{-i}\left(y_{-i}\right)\right)=E_{\left(\omega_{1}, \omega_{2}\right) \sim \lambda_{2}\left(b^{\prime} ; b^{\prime \prime} ; \sigma_{-i}\left(y_{-i}\right)\right)}\left[y_{i}^{\omega_{1}} \mid y_{i}, b, \sigma_{-i}\left(y_{-i}\right)\right] \\
& y_{i}\left(b^{\prime \prime} ; \sigma_{-i}\left(y_{-i}\right)\right)=E_{\left(\omega_{1}, \omega_{2}\right) \sim \lambda_{2}\left(b^{\prime} ; b^{\prime \prime} ; \sigma_{-i}\left(y_{-i}\right)\right)}\left[y_{i}^{\omega_{2}} \mid y_{i}, b, \sigma_{-i}\left(y_{-i}\right)\right]
\end{aligned}
$$


then the integrand in (8) can be written as

$$
E_{\left(\omega_{1}, \omega_{2}\right) \sim \lambda_{2}\left(b^{\prime} ; b^{\prime \prime} ; \sigma_{-i}\left(y_{-i}\right)\right)}\left[y_{i}^{\omega_{1} \vee \omega_{2}}+y_{i}^{\omega_{1} \wedge \omega_{2}}-y_{i}^{\omega_{1}}-y_{i}^{\omega_{2}} \mid y_{i}, b, \sigma_{-i}\left(y_{-i}\right)\right] .
$$

Because for every realization of $\left(\omega_{1}, \omega_{2}\right)$ from the distribution $\lambda_{2}\left(b^{\prime} ; b^{\prime \prime} ; \sigma_{-i}\left(y_{-i}\right)\right)$ we have

$$
y_{i}^{\omega_{1} \vee \omega_{2}}+y_{i}^{\omega_{1} \wedge \omega_{2}}-y_{i}^{\omega_{1}}-y_{i}^{\omega_{2}} \geq 0
$$

the expectation in (9) is also non-negative. Thus, for every $y_{-i}$, the integrand in (8) is non-negative. Therefore, (8) holds.

\section{Proof of Lemma 2}

Denote

$$
\begin{aligned}
b_{i}^{(0)} & =\left(b_{i 1}, b_{i 2}, b_{i 3}, \ldots, b_{i, L-1}, b_{i L}\right)^{T}, \\
b_{i}^{(1)} & =\left(\tilde{b}_{i 1}, b_{i 2}, b_{i 3}, \ldots, b_{i, L-1}, b_{i L}\right)^{T}, \\
b_{i}^{(2)} & =\left(\tilde{b}_{i 1}, \tilde{b}_{i 2}, b_{i 3}, \ldots, b_{i, L-1}, b_{i L}\right)^{T}, \\
& \cdots \\
b_{i}^{(L)} & =\left(\tilde{b}_{i 1}, \tilde{b}_{i 2}, \tilde{b}_{i 3}, \ldots, \tilde{b}_{i, L-1}, \tilde{b}_{i L}\right)^{T} .
\end{aligned}
$$

Represent the difference $\pi_{i}\left(\tilde{b}_{i} ; y_{i}, \sigma_{-i}\right)-\pi_{i}\left(b_{i} ; y_{i}, \sigma_{-i}\right)$ in the following way:

$$
\pi_{i}\left(\tilde{b}_{i} ; y_{i}, \sigma_{-i}\right)-\pi_{i}\left(b_{i} ; y_{i}, \sigma_{-i}\right)=\sum_{l=1}^{L}\left(\pi_{i}\left(b_{i}^{(l)} ; y_{i}, \sigma_{-i}\right)-\pi_{i}\left(b_{i}^{(l-1)} ; y_{i}, \sigma_{-i}\right)\right)
$$

Now represent the interim payoff function $\pi_{i}\left(b, y_{i}, \sigma_{-i}\right)$ by applying what we refer to throughout this paper as a marginalization technique:

$$
\begin{array}{r}
\pi_{i}\left(b_{i}, y_{i}, \sigma_{-i}\right)=\sum_{\omega: \omega_{l}=0} y_{i}^{\omega}\left(P_{i}^{\omega}\left(b_{i}, \sigma_{-i}\right)+P_{i}^{\omega \vee e_{l}}\left(b_{i}, \sigma_{-i}\right)\right)+\sum_{\omega: \omega_{l}=0}\left(y_{i}^{\omega \vee e_{l}}-y_{i}^{\omega}\right) P_{i}^{\omega \vee e_{l}}\left(b_{i}, \sigma_{-i}\right) \\
-\sum_{m=1}^{L} c_{i m}\left(b_{i m}, \sigma_{-i}\right) .
\end{array}
$$

Note that if $\omega_{l}=0$, then $P_{i}^{\omega}\left(b ; \sigma_{-i}\right)+P_{i}^{\omega \vee e_{l}}\left(b ; \sigma_{-i}\right)$ is the probability of winning all objects $m \neq l$ for which $\omega_{m}=1$ and losing all objects $m \neq l$ for which $\omega_{m}=0$. Thus, this is the probability of the event that does not depend on the allocation of object $l$ (also using Assumption 1). Therefore, $P_{i}^{\omega}\left(b ; \sigma_{-i}\right)+P_{i}^{\omega \vee e_{l}}\left(b ; \sigma_{-i}\right)$ does not depend on the bid for object $l$. Therefore, if $\omega_{l}=0$, then

$$
P_{i}^{\omega}\left(b_{i}^{(l)} ; \sigma_{-i}\right)+P_{i}^{\omega \vee e_{l}}\left(b_{i}^{(l)} ; \sigma_{-i}\right)-P_{i}^{\omega}\left(b_{i}^{(l-1)} ; \sigma_{-i}\right)-P_{i}^{\omega \vee e_{l}}\left(b_{i}^{(l-1)} ; \sigma_{-i}\right)=0
$$


because bid vectors $b_{i}^{(l)}$ and $b_{i}^{(l-1)}$ differ only in the bid submitted for object $l$. Hence,

$$
\begin{gathered}
\pi_{i}\left(b_{i}^{(l)} ; y_{i}, \sigma_{-i}\right)-\pi_{i}\left(b_{i}^{(l-1)} ; y_{i}, \sigma_{-i}\right)=\sum_{\omega: \omega_{l}=0}\left(y_{i}^{\omega \vee e_{l}}-y_{i}^{\omega}\right)\left(P_{i}^{\omega \vee e_{l}}\left(b_{i}^{(l)}, \sigma_{-i}\right)-P_{i}^{\omega \vee e_{l}}\left(b_{i}^{(l-1)}, \sigma_{-i}\right)\right) \\
-c_{i l}\left(\tilde{b}_{i l}, \sigma_{-i}\right)+c_{i l}\left(b_{i l}, \sigma_{-i}\right) .
\end{gathered}
$$

Note that because the component corresponding to the bid submitted for object $l$ in the vector $b_{i}^{(l)}$ is greater than the one in the vector $b_{i}^{(l-1)}$, and all the other components in the two vectors are the same, then

$$
\forall \omega: \omega_{l}=0 \quad P_{i}^{\omega \vee e_{l}}\left(b_{i}^{(l)}, \sigma_{-i}\right)-P_{i}^{\omega \vee e_{l}}\left(b_{i}^{(l-1)}, \sigma_{-i}\right) \geq 0 .
$$

Combining this across all objects $l$, we have

$$
\begin{aligned}
\pi_{i}\left(\tilde{b}_{i} ; y_{i}, \sigma_{-i}\right)-\pi_{i}\left(b_{i} ; y_{i}, \sigma_{-i}\right)=\sum_{l=1}^{L} \sum_{\omega: \omega_{l}=0}\left(y_{i}^{\omega \vee e_{l}}-y_{i}^{\omega}\right) & \underbrace{\left(P_{i}^{\omega \vee e_{l}}\left(b_{i}^{(l)}, \sigma_{-i}\right)-P_{i}^{\omega \vee e_{l}}\left(b_{i}^{(l-1)}, \sigma_{-i}\right)\right)}_{\geq 0} \\
& -\sum_{l=1}^{L} c_{i l}\left(\tilde{b}_{i l}, \sigma_{-i}\right)+\sum_{l=1}^{L} c_{i l}\left(b_{i l}, \sigma_{-i}\right) .
\end{aligned}
$$

The analogous representation for type $\tilde{y}_{i}$ is

$$
\begin{aligned}
\pi_{i}\left(\tilde{b}_{i} ; \tilde{y}_{i}, \sigma_{-i}\right)-\pi_{i}\left(b_{i} ; \tilde{y}_{i}, \sigma_{-i}\right)=\sum_{l=1}^{L} \sum_{\omega: \omega_{l}=0}\left(\tilde{y}_{i}^{\omega \vee e_{l}}-\tilde{y}_{i}^{\omega}\right) & \underbrace{\left(P_{i}^{\omega \vee e_{l}}\left(b_{i}^{(l)}, \sigma_{-i}\right)-P_{i}^{\omega \vee e_{l}}\left(b_{i}^{(l-1)}, \sigma_{-i}\right)\right)}_{\geq 0} \\
& -\sum_{l=1}^{L} c_{i l}\left(\tilde{b}_{i l}, \sigma_{-i}\right)+\sum_{l=1}^{L} c_{i l}\left(b_{i l}, \sigma_{-i}\right) .
\end{aligned}
$$

From the definition of the partial order we then conclude that

$$
\pi_{i}\left(\tilde{b}_{i} ; \tilde{y}_{i}, \sigma_{-i}\right)-\pi_{i}\left(b_{i} ; \tilde{y}_{i}, \sigma_{-i}\right) \geq \pi_{i}\left(\tilde{b}_{i} ; y_{i}, \sigma_{-i}\right)-\pi_{i}\left(b_{i} ; y_{i}, \sigma_{-i}\right),
$$

which implies the weak single crossing property.

\section{Proof of Proposition 2}

Let $\mu_{2^{L}-1}$ denote the Lebesgue measure in $\mathbb{R}^{2^{L}-1}$. Fix any $S \subset \mathcal{Y}_{i}$ with a positive $F_{Y_{i}}$ measure. By the absolute continuity assumption then, $\mu_{2^{L}-1}(S)>0$. We start by showing that we can find two points in $S$-denote them as $\bar{y}_{i}$ and $\tilde{y}_{i},-$ such that all the inequalities in the definition of the partial order will be satisfied strictly. In other words, we want to find $\bar{y}_{i}, \tilde{y}_{i} \in S$ such that $\tilde{y}_{i} \in \bar{y}_{i}+\mathcal{Z}_{2^{L}-1}^{o}$. Set $\mathcal{Z}_{2^{L}-1}^{o}$ is the interior in $\mathbb{R}^{2^{L}-1}$ of cone $\mathcal{Z}_{2^{L}-1}$ defined in (2) (it is discussed in the main text of the paper that $\mathcal{Z}_{2^{L}-1}^{o} \neq \emptyset$ ). 
We claim that we can take any $\bar{y}_{i} \in S$ that is a point of (Lebesgue) density of $S$. The property of being a point of density of $S$ means that

$$
\lim _{r \downarrow 0} \frac{\mu_{2^{L}-1}\left(\left(\bar{y}_{i}+\mathbf{B}_{r}\right) \cap S\right)}{\mu_{2^{L}-1}\left(\bar{y}_{i}+\mathbf{B}_{r}\right)}=1
$$

where $\mathbf{B}_{r}$ denotes the open ball in $\mathbb{R}^{2^{L}-1}$ centered at 0 of radius $r>0$.

Note that if $\bar{y}_{i} \in S$ is a point of density of $S$ and the set $\left(y_{i}+\mathcal{Z}\right) \cap S$ is empty, then

$$
\begin{aligned}
\frac{\mu_{2^{L}-1}\left(\left(\bar{y}_{i}+\mathbf{B}_{r}\right) \cap S\right)}{\mu_{2^{L}-1}\left(\bar{y}_{i}+\mathbf{B}_{r}\right)} & =\frac{\mu_{2^{L}-1}\left(\left(\bar{y}_{i}+\mathbf{B}_{r}\right) \cap S \cap\left(\bar{y}_{i}+\mathcal{Z}_{2^{L}-1}^{o}\right)^{c}\right)}{\mu_{2^{L}-1}\left(\bar{y}_{i}+\mathbf{B}_{r}\right)} \\
& \leq \frac{\mu_{2^{L}-1}\left(\left(\bar{y}_{i}+\mathbf{B}_{r}\right) \cap\left(\bar{y}_{i}+\mathcal{Z}_{2^{L}-1}^{o}\right)^{c}\right)}{\mu_{2^{L}-1}\left(\bar{y}_{i}+\mathbf{B}_{r}\right)}
\end{aligned}
$$

for all $r>0$. Note that the ratio

$$
\frac{\mu_{2^{L}-1}\left(\mathcal{Z}_{2^{L}-1}^{o} \cap \mathbf{B}_{r}\right)}{\mu_{2^{L}-1}\left(\mathbf{B}_{r}\right)}
$$

is strictly between zero and one does not depend on $r .{ }^{16}$ Denote this ratio as $c_{L} \in(0,1)$. Using the translation invariance property of the Lebesgue measure we obtain then that

$$
\frac{\mu_{2^{L}-1}\left(\left(\bar{y}_{i}+\mathbf{B}_{r}\right) \cap S\right)}{\mu_{2^{L}-1}\left(\bar{y}_{i}+\mathbf{B}_{r}\right)} \leq \frac{\mu_{2^{L}-1}\left(\left(\bar{y}_{i}+\mathbf{B}_{r}\right) \cap\left(\bar{y}_{i}+\mathcal{Z}_{2^{L}-1}^{o}\right)^{c}\right)}{\mu_{2^{L}-1}\left(\bar{y}_{i}+\mathbf{B}_{r}\right)}=1-c_{L}
$$

for all $r>0$, but this contradicts the property of $\bar{y}_{i}$ being a point of density of $S$. This contradiction means that for any point $\bar{y}_{i}$ of Lebesgue density of $S$, we can find $\tilde{y}_{i} \in$ $\bar{y}_{i}+\mathcal{Z}_{2^{L}-1}^{O}$ to give us the conclusion. By the Lebesgue property a.e. $\bar{y}_{i} \in S$ is a point of density.

By convexity, the boundary of each set in the finite union of sets composing $\mathcal{Y}_{i}$ has Lebesgue measure zero in $\mathbb{R}^{2^{L}-1}$. Since the boundary of a finite union of sets is contained in the finite union of the respective boundaries, we conclude that the boundary of $\mathcal{Y}_{i}$ has Lebesgue measure zero in $\mathbb{R}^{2^{L}-1}$. Therefore, without loss of generality, we can select both $y_{i} \in S$ and $\tilde{y}_{i} \in S \cap\left(y_{i}+\mathcal{Z}_{2^{L}-1}^{o}\right)$ to be in the interior of $\mathcal{Y}_{i}$ in $\mathbb{R}^{2^{L}-1}$. Therefore, we can find a small $r>0$ such that the ball $\tilde{y}_{i}+\mathbf{B}_{r}$ is contained in $\left(y_{i}+\mathcal{Z}_{2^{L}-1}^{o}\right) \cap \mathcal{Y}_{i}$. Consider the intersection $\left(\tilde{y}_{i}+\mathbf{B}_{r}\right) \cap\left(\tilde{y}_{i}+\mathcal{Z}_{2^{L}-1}^{o}\right)$. This intersection is non-empty and is an open set in $\mathbb{R}^{2^{L}-1}$. Every point in this intersection dominates $\bar{y}_{i}$ but is dominated by $\tilde{y}_{i}$ in the sense of partial order $(P O)$. As a union of a finite number of compact sets, $\mathcal{Y}_{i}$ is compact in $\mathbb{R}^{2^{L}-1}$ and, hence, has a dense countable subset. Denote it as $\mathcal{Y}_{i}^{\star}$. Set $\left(\tilde{y}_{i}+\mathbf{B}_{r}\right) \cap\left(\tilde{y}_{i}+\mathcal{Z}_{2^{L}-1}^{o}\right)$

\footnotetext{
${ }^{16}$ To see this, consider the scaling transform $y \rightarrow \frac{y}{r}$. It maps the $\mathcal{Z}_{2^{L}-1}^{o} \cap \mathbf{B}_{r}$ to $\mathcal{Z}_{2^{L}-1}^{o} \cap \mathbf{B}_{1}$. Since the Jacobian of this sort of transform is independent of $y$, the ratio of the volume of the intersection of shape $\mathcal{Z}_{2^{L}-1}^{o}$ with he ball $\mathbf{B}_{r}$ to the volume of $\mathbf{B}_{r}$ is invariant under such a transform.
} 
being a non-empty open set in $\mathbb{R}^{2^{L}-1}$ clearly has to contain a point from $\mathcal{Y}_{i}^{\star}$.

\section{Proof of Proposition 3}

We define the partial order $\succeq$ on the space of types as in $(P O)$. Let us define the partial order in each bid (= action) space as the coordinatewise order:

$$
\tilde{b}_{i} \succeq b_{i} \quad \Longleftrightarrow \quad \tilde{b}_{i} \geq b_{i}
$$

where $\tilde{b}_{i} \geq b_{i}$ means that each component on the $L$-dimensional vector $\tilde{b}_{i}$ is greater or equal than the corresponding component of $b_{i}$.

We verify conditions of Theorem 4.1 and Proposition 4.4 in Reny (2011). Let us start with conditions G1-G6 in Theorem 4.1.

G1 Obviously, the partial order given in Definition 4 is transitive:

$$
\tilde{\tilde{y}}_{i} \succeq \tilde{y}_{i}, \tilde{y}_{i} \succeq y_{i} \quad \Rightarrow \quad \tilde{\tilde{y}}_{i} \succeq y_{i}
$$

it is also reflexive:

$$
y_{i} \succeq y_{i}
$$

and it is also antisymmetric:

$$
\tilde{y}_{i} \succeq y_{i} \text { and } y_{i} \succeq \tilde{y}_{i} \quad \Rightarrow \quad \tilde{y}_{i}=y_{i} \text {. }
$$

Let $\mathcal{A}\left(\Re^{2^{L}} \cap \mathcal{Y}_{i}\right)$ denote the Borel sigma-field on $\Re^{2^{L}} \cap \mathcal{Y}_{i}$. Let us show that

$$
G_{i} \equiv\left\{\left(\tilde{y}_{i}, y_{i}\right) \in \mathcal{Y}_{i} \times \mathcal{Y}_{i}: \tilde{y}_{i} \succeq y_{i}\right\} \in \mathcal{A}\left(\Re^{2^{L}} \cap \mathcal{Y}_{i}\right) \times \mathcal{A}\left(\Re^{2^{L}} \cap \mathcal{Y}_{i}\right)
$$

Notice that the partial order between $\tilde{y}_{i}$ and $y_{i}$ can be fully expresses as comparison of certain linear inequalities for $\tilde{y}_{i}$ and $y_{i}$. In other words, the order $\tilde{y}_{i} \succeq y_{i}$ can be fully described as a system of linear inequalities

$$
M_{L} \tilde{y}_{i}-M_{L} y_{i} \geq 0
$$

where matrix $M_{L}$ is defined in a certain way. Therefore, $G_{i}$ is the intersection of a closed convex polyhedron in $\mathbb{R}^{2 \cdot 2^{L}}$ with $\mathcal{Y}_{i} \times \mathcal{Y}_{i}$, and thus, $G_{i} \in \mathcal{A}\left(\Re^{2 \cdot 2^{L}} \cap \mathcal{Y}_{i} \times \mathcal{Y}_{i}\right)$. Under the Euclidean metric $\Re^{2^{L}} \cap \mathcal{Y}_{i}$ is a locally compact Hausdorff space. Therefore, by e.g. Proposition 7.6.2 on page 220 in Cohn (2013),

$$
\mathcal{A}\left(\Re^{2 \cdot 2^{L}} \cap \mathcal{Y}_{i} \times \mathcal{Y}_{i}\right)=\mathcal{A}\left(\Re^{2^{L}} \cap \mathcal{Y}_{i}\right) \times \mathcal{A}\left(\Re^{2^{L}} \cap \mathcal{Y}_{i}\right),
$$

and, hence, $G_{i} \in \mathcal{A}\left(\Re^{2^{L}} \cap \mathcal{Y}_{i}\right) \times \mathcal{A}\left(\Re^{2^{L}} \cap \mathcal{Y}_{i}\right)$.

G2 The atomlessness of the measure on the sigma-algebra for $\mathcal{Y}_{i}$ follows from the continuity of c.d.f. $F_{Y, i}$ in Assumption 3. 
G3 This is assumed explicitly.

G4 $\mathcal{B}_{i}$ is assumed to be a lattice. Also,

$$
\left\{\left(\tilde{b}_{i}, b_{i}\right) \in \mathcal{B}_{i} \times \mathcal{B}_{i}: \tilde{b}_{i} \succeq b_{i}\right\}
$$

is closed in the product topology on $\mathbb{R}^{L} \times \mathbb{R}^{L}$ because the partial order on $\mathcal{B}_{i}$ is the coordinate-wise partial order.

G5 Each $\mathcal{B}_{i}$ is a locally-complete Euclidean metric lattice.

G6 The function

$$
\begin{array}{r}
u_{i}\left(b_{i}, b_{-i} ; y_{i}\right)=\sum_{\omega}\left(y_{i}^{\omega}-\omega^{\prime} b_{i}\right) \cdot \prod_{l: \omega^{l}=1}\left(1\left(b_{i l}>\max _{j \neq i} b_{j l}\right)+\right. \\
\left.\times T_{i l}\left(S_{l}, b_{i l}\right) 1\left(b_{i l}=\max _{j \neq i} b_{j l}\right)\right) \\
\times \prod_{l: \omega^{l}=0} 1\left(b_{i l}<\max _{j \neq i} b_{j l}\right)
\end{array}
$$

is jointly measurable. It is bounded since both $\mathcal{Y}_{i}$ and $\mathcal{B}_{i}$ are bounded. Note that $T_{i l}\left(S_{l}, b_{i l}\right)$, where $S_{l}$ denotes the set of bidders tying for the highest bid in auction $l$, stands for the probability of obtaining object $l$ in case of ties. This formulation accords with Assumption 1.

The function $u_{i}\left(b_{i} ; y_{i}\right)$ is also continuous in $b_{i} \in \mathcal{B}_{i}$ for every $y_{i} \in \mathcal{Y}_{i}$ since by assumption $\mathcal{B}_{i}$ consists of a finite number of points.

Thus, G1-G6 in Theorem 4.1 in Reny (2011) hold. Clearly, Lemmas 1 and 2 of this paper imply (i) in Proposition 4.4 in Reny (2011).

\section{Proof of Lemma 3}

Fix $\epsilon>0$. Choose $k$ such that $\delta_{k} \equiv \max _{j=0, \ldots, N} \mathcal{H}\left(\ddot{\mathcal{B}}_{j}^{k}, \mathcal{B}_{j}\right)<\epsilon / L$.

For local bidder $j=1, \ldots, N$ with type realization $y_{j} \in \mathcal{Y}_{j}$, the argument is entirely standard; we reproduce it here only for completeness. Let $\pi_{j}^{*}=\max _{b_{j} \in \mathcal{B}_{j}} \pi_{j}\left(y_{j}, b_{j} ; \tilde{\sigma}_{-j}^{k}\right)$ and $b_{j}^{*}=\arg \max _{b_{j} \in \mathcal{B}_{j}} \pi_{j}\left(y_{j}, b ; \tilde{\sigma}_{-j}^{k}\right)$, and let $\ddot{b}_{j}$ be the smallest element of $\ddot{\mathcal{B}}_{j}$ such that $\ddot{b}_{j} \geq b_{j}^{*}$. Clearly, we must have $b_{j}^{*} \leq y_{j}$, hence the loss associated with bidding $\ddot{b}_{j}$ (rather than $b_{j}^{*}$ ) can be no greater than $\delta_{k}$. But $\ddot{b}_{j}$ was feasible for bidder $j$ under grid space $\ddot{\mathcal{B}}^{k}$, whence by equilibrium $\pi_{j}\left(y_{j}, \ddot{\sigma}_{j}^{k}\left(y_{j}\right) ; \ddot{\sigma}_{-j}^{k}\right) \geq \pi_{j}\left(y_{j}, \bar{b}_{j} ; \ddot{\sigma}_{-j}^{k}\right) \geq \pi_{k}^{*}-\delta_{k}>\pi_{k}^{*}-\epsilon$. Since $y_{j}$ was arbitrary, this bound also applies in expectation. Thus local bidder $j$ can gain no more than $\epsilon$ by deviating from strategy profile $\ddot{\sigma}_{j}^{k}$ when rivals play strategy profile $\ddot{\sigma}_{-j}^{k}$; i.e. $\ddot{\sigma}_{-j}^{k}$ satisfies the conditions of an $\epsilon$-equilibrium for local bidder $j$.

For the global bidder 0, the argument is somewhat more subtle, turning on the fact that action spaces for bidder 0 are always such that bidder 0 can choose (at cost no greater than the bid increment) whether and how to resolve potential ties. Fixing type $y_{0} \in \mathcal{Y}_{0}$ and holding strategies of local bidders fixed at $\ddot{\sigma}_{-0}^{k}$, let $\pi_{0}^{*}$ denote bidder 0 's supremum payoff 
over the unrestricted bid space $\mathcal{B}_{0}$. Let $\left\{b_{0}^{m}\right\}_{m=1}^{\infty}$ be any sequence of $L \times 1$ bid vectors for bidder 0 such that $\pi_{0}\left(y_{0}, b_{0}^{m} ; \ddot{\sigma}_{-0}^{k}\right) \rightarrow \pi_{0}^{*}$ as $m \rightarrow 0$. Let $\left\{b_{0 l}^{m}\right\}_{m=1}^{\infty}$ be the scalar sequence such that for each $m=1,2, \ldots$ the scalar $b_{0 l}^{m}$ is the $l$ th element of the $L \times 1$ bid vector $b_{0}^{m}$; i.e. the element describing bidder 0 's bid in auction $l$. Construct a sequence $\left\{\ddot{b}_{0 l}^{m}\right\}_{m=1}^{\infty}$ from $\left\{b_{0 l}^{m}\right\}_{m=1}^{\infty}$ as follows: if $b_{0 l}^{m} \in \ddot{\mathcal{B}}_{l}^{k}$, then $\ddot{b}_{0 l}^{m}=b_{0 l}^{m}$, otherwise

$$
\ddot{b}_{0 l}^{m}=\min \left\{b_{l}^{\prime} \in \ddot{\mathcal{B}}_{0 l}^{k}: b_{l}^{\prime}>\max \left\{b_{l}^{\prime \prime} \in \ddot{\mathcal{B}}_{l}^{k} \mid b_{l}^{\prime \prime}<b_{0 l}^{m}\right\}\right\} .
$$

Recall that $\ddot{\mathcal{B}}_{l}^{k}$ is a selection from $\ddot{\mathcal{B}}_{0 l}^{k}$ such that for any two points $b_{l}^{\prime}, b_{l}^{\prime \prime}$ in $\ddot{\mathcal{B}}_{l}^{k}$ there exists a third point $b_{l}^{\prime \prime \prime} \in \ddot{\mathcal{B}}_{0 l}^{k} \backslash \ddot{\mathcal{B}}_{l}^{k}$ such that $b_{l}^{\prime}<b_{l}^{\prime \prime \prime}<b_{l}^{\prime \prime}$. Furthermore, from the perspective of bidder 0 , allocation probabilities for each $l$ are flat outside the support $\ddot{\mathcal{B}}_{l}^{k}$ of rival strategies $\ddot{\sigma}_{-0}^{k}$. Hence by construction the sequence $\ddot{b}_{0 l}^{m}$ will yield the same allocation probabilities as the sequence $b_{0 l}^{m}$ for any $m=1,2, \ldots$. Thus bidder 0 's expected payoffs along bid sequence $\ddot{b}_{0 l}^{m}$ can differ from bidder 0's expected payoffs along bid sequence $b_{0 l}^{m}$ by at most the bid increment $\delta_{k}$. Repeating this construction separately for each $l=1, \ldots, L$, we obtain a sequence $\left\{\ddot{b}_{0}^{m}\right\}_{m=1}^{\infty}$ contained in $\ddot{\mathcal{B}}_{0}^{k}$, yielding the same allocations as $\left\{b_{0}^{m}\right\}_{m=1}^{\infty}$, and for which bidder 0's payoffs differ (relative to $\left\{b_{0}^{m}\right\}_{m=1}^{\infty}$ ) by at most $L \delta_{k}$. But (by equilibrium) bidder 0's payoffs under the equilibrium strategy $\ddot{\sigma}_{0}^{k}$ must be weakly greater than bidder 0's payoffs at each feasible deviation, and $\left\{\ddot{b}_{0}^{m}\right\}_{m=1}^{\infty}$ is a sequence of feasible deviations which eventually attain a payoff within $L \delta_{k}<\epsilon$ of bidder 0's unrestricted supremum at type $y_{0}$. Since $y_{0}$ was arbitrary, this bound on bidder 0's interim gains also implies a bound on bidder 0's ex ante gains, whence it follows that $\ddot{\sigma}^{k}$ is an $\epsilon$-equilibrium on the unrestricted bid space $\mathcal{B}$.

\section{Proof of Lemma 4}

Let $(\tilde{\sigma}, \tilde{\Pi})$ be any point in the closure of the vector payoff function

$$
\Pi(\sigma)=\left(\Pi_{0}(\sigma) ; \Pi_{1}(\sigma), \ldots, \Pi_{N}(\sigma)\right),
$$

i.e. any $\tilde{\Pi}$ for which there exists a sequence of monotone strategies $\left\{\sigma^{k}\right\}_{k=1}^{\infty}$ such that $\sigma^{k} \rightarrow \tilde{\sigma}$ (under the metric of Lemma A.13 in Reny (2011)) and $\Pi\left(\sigma^{k}\right) \rightarrow \tilde{\Pi}$. Suppose that $\tilde{\sigma}$ is not an equilibrium. We need to show that some player $i \in\{0,1, \ldots, N\}$ can secure a payoff strictly above $\tilde{\Pi}_{i}$ at $\tilde{\sigma}$.

First suppose that under the limiting strategy $\tilde{\sigma}$ relevant ties occur with probability zero. The vector ex ante payoff function $\Pi(\cdot)$ is then continuous in a neighborhood of $\tilde{\sigma}$, from which it follows that $\tilde{\boldsymbol{\Pi}}=\boldsymbol{\Pi}(\tilde{\sigma})$. By hypothesis $\tilde{\sigma}$ is not an equilibrium, hence there exists a player $i$ and a strategy $\sigma_{i}^{0}$ such that

$$
\Pi_{i}\left(\sigma_{i}^{0}, \tilde{\sigma}_{-i}\right)>\Pi_{i}\left(\tilde{\sigma}_{i} ; \tilde{\sigma}_{-i}\right)
$$

Let $\Pi_{i}^{*}$ be $i$ 's supremum payoff against rival strategies $\tilde{\sigma}_{-i}$, and let $\left\{\sigma_{i}^{n}\right\}_{n=1}^{\infty}$ be any sequence of strategies for $i$ such that $\Pi_{i}\left(\sigma_{i}^{n} ; \tilde{\sigma}_{-i}\right) \rightarrow \Pi_{i}^{*}$. Recall that ties are broken randomly across bidders and auctions. Hence if relevant ties occur with probability bounded away from zero along the sequence $\left\{\sigma^{n}\right\}_{n=1}^{\infty}$, it must be that $i$ is indifferent as to how these are 
resolved. ${ }^{17}$ This in turn implies that $i$ 's supremum payoff $\Pi_{i}^{*}$ can also be approached by a strategy sequence $\left\{\sigma_{i}^{n}\right\}_{n=1}^{\infty}$ such that for each $n$ the random vector $B_{i}=\sigma_{i}^{n}\left(Y_{i}\right)$ has a joint distribution with atomless marginals. ${ }^{18}$ Choose any such sequence. Since $\Pi_{i}^{*} \geq \Pi_{i}^{0}>\tilde{\Pi}_{i}$ there will exist an $n$ such that

$$
\Pi_{i}\left(\sigma_{i}^{n} ; \tilde{\sigma}_{-i}\right)>\tilde{\Pi}_{i}
$$

But by construction $\sigma_{i}^{n}$ generates a joint bid distribution with atomless marginals, hence there is no rival strategy profile under which $i$ ties with positive probability under $\sigma_{i}^{n}$. Thus $\Pi_{i}\left(\sigma_{i}^{n} ; \cdot\right)$ is also continuous in $\sigma_{-i}$ at $\sigma_{i}^{n}$ for each $n$. Hence for large enough $n$, we will have

$$
\Pi_{i}\left(\sigma_{i}^{n} ; \sigma_{-i}\right)>\tilde{\Pi}_{i}
$$

for all $\sigma_{-i}$ in a neighborhood of $\tilde{\sigma}_{-i}$. Thus bidder $i$ can secure a payoff strictly above $\tilde{\Pi}_{i}$.

Now suppose that under the limiting strategy $\tilde{\sigma}$, a relevant tie occurs with positive probability for at least one auction $l=1, \ldots, L$. In particular, suppose that such a tie occurs at bid $\bar{b}_{l}$. By construction, this tie must involve at least one local bidder $i$. Let $\tilde{Y}_{i}\left(\bar{b}_{l}\right)$ be the set of types such that $i$ bids $\bar{b}_{l}$ under the limiting strategy $\tilde{\sigma}$; note that $\tilde{Y}_{i}\left(\bar{b}_{l}\right)$ has positive measure by construction. Clearly we must have $v_{i} \geq \bar{b}_{l}$ almost everywhere in $\tilde{Y}_{i}\left(\bar{b}_{l}\right)$, since otherwise $i$ could strictly gain by deviating to a bid strictly below $\bar{b}_{l}$ on a relevant subset of types. Furthermore, we can have $v_{i}=\bar{b}_{l}$ on at most a measure- 0 subset of $\tilde{Y}_{i}\left(\bar{b}_{l}\right)$. Hence we must have $v_{i}>\bar{b}_{l}$ almost everywhere in $\tilde{Y}_{i}\left(\bar{b}_{l}\right)$. But in this case either $i$ wins with probability approaching 1 against any other rival type submitting limiting bid $\bar{b}_{l}$ along the sequence $\sigma^{k}$, or $i$ can secure a payoff strictly above $\tilde{\Pi}_{i}$ by deviating on $\tilde{Y}_{i}\left(\bar{b}_{l}\right)$ to a bid infinitesimally above $\bar{b}_{l}$.

Thus for any sequence of strategies converging to a tie between two local bidders, or for any sequence of strategies converging to a tie between the global bidder and one local bidder such that tying types of the local bidder lose with probability bounded away from zero along the sequence, at least one player $i$ can secure a payoff strictly greater than $\tilde{\Pi}_{i}$. But one potential problem case still remains: a sequence of strategies $\sigma^{k}$ converging to a relevant tie between the global bidder 0 and a single local bidder $i$, in which almost every type of bidder 0 tying at $\bar{b}_{l}$ in the limit loses against almost every type of bidder $i$ tying at $\bar{b}_{l}$ in the limit with probability approaching one along the sequence. We will show that the global bidder can secure a payoff strictly above $\tilde{\Pi}_{0}$ in this case.

Toward this end, suppose that $\sigma^{k}$ is a sequence satisfying the description above. Consider type $y \in \mathcal{Y}_{0}$ of bidder 0. Following Gentry, Komarova and Schiraldi (2019), Let the $L \times 1$ vector $V(y)$ denote bidder 0's standalone valuations for winning each object $\ell=1, \ldots, L$ individually; i.e. $V_{\ell}(y)=y^{e_{\ell}}$ for all $\ell$. Similarly, let $K(y)$ denote the $2^{L}-1 \times 1$ vector given by

$$
K(y)=y-\Omega V(y)
$$

\footnotetext{
${ }^{17}$ For local bidders, one can easily rule out ties along $\left\{\sigma^{n}\right\}_{n=1}^{\infty}$; for global bidders, the argument is more complicated, so we temporarily allow them.

${ }^{18}$ For instance, one could add a small continuously distributed error $\epsilon$ to each bid, with the support of $\epsilon$ tending to a unit mass at 0 and the sign of $\epsilon$ adjusted as needed to ensure $i$ 's preferred tie-breaking resolution at each type $y_{i}$.
} 
where $\Omega$ denotes the $2^{L}-1 \times L$ outcome matrix defined in Section 2 . We refer to $K^{\omega}(y)$ as the complementarity that type $y$ of bidder 0 associates with each combination $\omega$; that is, the difference between $i$ 's value $y^{\omega}$ for the combination and the sum of $i$ 's standalone valuations for each object won in the combination.

Let $\bar{Y}_{0 l}$ be the set of $y \in \mathcal{Y}_{0}$ such that $\tilde{\sigma}_{0 l}=\bar{b}_{l}$. For each $l=1, \ldots, L$, define $\Gamma_{l}\left(y ; \sigma^{k}\right)$ as the marginal probability that bidder 0 with type $y \in \mathcal{Y}_{0}$ wins auction $l=1$ under strategy profile $\sigma^{k}$, and let $\tilde{\Gamma}_{l}(y)=\lim _{k \rightarrow \infty} \Gamma_{l}\left(y ; \sigma^{k}\right) .{ }^{19}$ Since independence of types across local rivals implies independence of bids across both rivals and auctions, we may then write the limiting interim payoff of bidder 0 at type $y$ as follows:

$$
\begin{aligned}
\tilde{\pi}(y) & =\tilde{\Gamma}_{l}(y)\left[V_{l}(y)-\bar{b}_{l}\right]+\sum_{\ell \neq l} \tilde{\Gamma}_{\ell}(y)\left[V_{\ell}(y)-\tilde{\sigma}_{0 \ell}(y)\right]+\sum_{\omega} K^{\omega}(y) \prod_{\ell=1}^{L} \tilde{\Gamma}_{\ell}(y)^{\omega_{\ell}}\left[1-\tilde{\Gamma}_{\ell}(y)\right]^{1-\omega_{\ell}} \\
& =\sum_{\ell \neq l} \tilde{\Gamma}_{\ell}(y)\left[V_{\ell}(y)-\tilde{\sigma}_{0 \ell}(y)\right]+\left\{\sum_{\omega: \omega_{l}=0} K^{\omega}(y) \prod_{\ell \neq l} \tilde{\Gamma}_{\ell}(y)^{\omega_{\ell}}\left[1-\tilde{\Gamma}_{\ell}(y)\right]^{1-\omega_{\ell}}\right\} \\
& +\tilde{\Gamma}_{l}(y) \cdot\left\{\left[V_{l}(y)-\bar{b}_{l}\right]+\sum_{\omega: \omega_{l}=0}\left[K^{\omega \vee e_{l}}(y)-K^{\omega}(y)\right] \prod_{\ell \neq l} \tilde{\Gamma}_{\ell}(y)^{\omega_{\ell}}\left[1-\tilde{\Gamma}_{\ell}(y)\right]^{1-\omega_{\ell}}\right\},(11)
\end{aligned}
$$

where we obtain the second expression from the first by partitioning the set of outcomes $\omega$ into those with $\omega_{\ell}=0$ and those with $\omega_{\ell}=1$, factoring out terms involving $\tilde{\Gamma}_{l}(y)$ from the joint win probability $\prod_{\ell} \tilde{\Gamma}_{\ell}(y)^{\omega_{\ell}}\left[1-\tilde{\Gamma}_{\ell}(y)\right]^{1-\omega_{\ell}}$, and recombining terms, noting that

$$
\left\{\omega: \omega_{l}=1\right\}=\left\{\omega \vee e_{l} \text { for } \omega: \omega_{l}=0\right\} .
$$

For each auction $l$, let $\underline{v}_{l}$ denote the infimum of the support of $V_{l}\left(Y_{0}\right)$. Restrict attention to the subset of $y$ in $\overline{\mathcal{Y}}_{0 l}$ such that $V_{l}(y)>\underline{v}_{l}$; since $V_{l}(y)=\underline{v}_{l}$ for at most a set of $F_{0}$-measure 0 in $\mathcal{Y}_{0}$, this subset has the same $F_{0}$-measure as $\overline{\mathcal{Y}}_{0 l}$. Suppressing $y$ in notation, we may write the final term in braces as follows:

$$
\left(V_{l}-\bar{b}_{l}\right)+\sum_{\omega: \omega_{l}=0}\left[K^{\omega \vee e_{l}}-K^{\omega}\right] \prod_{\ell \neq l} \tilde{\Gamma}_{\ell}^{\omega_{\ell}}\left[1-\tilde{\Gamma}_{\ell}\right]^{1-\omega_{\ell}} .
$$

Note that this term reflects bidder 0's net expected marginal valuation for winning auction $l$, taking as given her expected probabilities of winning other auctions $\ell \neq l$ (which, critically, do not depend on $b_{l}$ ).

First suppose that the expected marginal valuation (12) above is strictly positive for $F_{0}$-a.e. $y \in \overline{\mathcal{Y}}_{0 l}$. Then bidder 0 can secure a payoff strictly above $\tilde{\Pi}_{0}$ by deviating at each $y \in \overline{\mathcal{Y}}_{0 l}$ to a bid $b_{l}^{\prime}$ slightly greater than $\bar{b}_{l}$.

Now suppose instead that the marginal valuation (12) is nonpositive on a subset of $\overline{\mathcal{Y}}_{0 l}$ with positive $F_{0}$-measure. The supermodularity of complementarities implies $K^{\omega \vee e_{l}} \geq K^{\omega}$

\footnotetext{
${ }^{19}$ Note that by construction we have $\tilde{\Gamma}_{l}(y) \neq \Gamma_{l}(y ; \tilde{\sigma})$ for $y \in \overline{\mathcal{Y}}_{0 l}$.
} 
for all $\omega$, whence

$$
V_{l}+\sum_{\omega: \omega_{l}=0}\left[K^{\omega \vee e_{l}}-K^{\omega}\right] \prod_{\ell \neq l} \tilde{\Gamma}_{\ell}^{\omega_{\ell}}\left[1-\tilde{\Gamma}_{\ell}\right]^{1-\omega_{\ell}}>0
$$

Recall that, by hypothesis, $\underline{v}_{l}$ is weakly greater than the infimum of the support of $Y_{i}$ for each local rival $i$ with $\ell_{i}=l$. Hence, when local rivals employ undominated strategies, any $b_{l}>\underline{v}_{l}$ implies a strictly positive probability of winning auction $l$. Hence there exists a $b_{l}^{\prime} \in\left(\underline{v}_{l}, V_{l}\right)$ for which both $\tilde{\Gamma}_{l}^{\prime}>0$ and

$$
V_{l}-b_{l}^{\prime}+\sum_{\omega: \omega_{l}=0}\left[K^{\omega \vee e_{l}}-K^{\omega}\right] \prod_{\ell \neq l} \tilde{\Gamma}_{\ell}^{\omega_{\ell}}\left[1-\tilde{\Gamma}_{\ell}\right]^{1-\omega_{\ell}}>0 .
$$

The first line of (11) does not depend on $\tilde{\Gamma}_{l}$, so the only effect of deviating to bid $b_{l}^{\prime}$ at type $y$ is to replace the nonpositive final term in the limiting payoff (11) with a strictly positive limiting term. By construction, this represents a strict increase in limiting payoffs, which bidder 0 may secure against any sequence of rival strategies converging to $\tilde{\sigma}_{-0}$ by infinitesimally increasing the deviating bid $b_{l}^{\prime}$. Furthermore, by hypothesis, such securing deviations exist on a set of types $y \in \overline{\mathcal{Y}}_{0}$ of positive $F_{0}$-measure. Hence in this case bidder 0 can secure an ex ante payoff strictly greater than $\tilde{\Pi}_{0}$.

Taken together, the cases above establish that for any point $(\tilde{\sigma}, \tilde{\boldsymbol{\Pi}})$ in the closure of the graph of the vector ex ante payoff function $\Pi(\cdot)$, if $\tilde{\sigma}$ is not an equilibrium then at least one player $i \in\{0,1, \ldots, N\}$ can secure a payoff strictly above $\tilde{\Pi}_{i}$. This is what was to be shown.

\section{Proof of Proposition 5}

The proof of Proposition 5 is by construction.

Consider any sequence $\left\{\ddot{\mathcal{B}}_{1}^{k}, \ldots, \ddot{\mathcal{B}}_{L}^{k}\right\}_{k=1}^{\infty}$ of finite bid lattices for auctions $l=1, \ldots L$, such that $\mathcal{H}\left(\ddot{\mathcal{B}}_{l}^{k}, \mathcal{B}_{l}\right) \rightarrow 0$ for all $l$, where $\mathcal{H}$ denotes Hausdorff distance. For each $k=1,2, \ldots$, let the bid space for bidder $i=1, \ldots, N, \ddot{\mathcal{B}}_{i}^{k}$, be the Cartesian product of bid spaces $\ddot{\mathcal{B}}_{l}^{k}$ for the auctions $l$ in which $i$ is competing, and let $\ddot{\mathcal{B}}^{k}=\times_{i=1}^{N} \ddot{\mathcal{B}}_{i}^{k}$ denote the Cartesian product of action spaces for all bidders. Proposition 3 implies that for each $k$ there exists a monotone pure strategy bidding equilibrium $\sigma^{k}$ on $\ddot{\mathcal{B}}^{k}$, where as usual monotonicity is understood in the sense of the partial order $(P O)$ on types and the coordinatewise order on bids. As in Section 5.1, we may invoke Lemma A.13 in Reny (2011) to conclude that the space of strategies monotone with respect to $(P O)$ is compact, and hence that the sequence $\left\{\ddot{\sigma}^{k}\right\}_{k=1}^{\infty}$ has a subsequence $\left\{\ddot{\sigma}^{k_{j}}\right\}_{j=1}^{\infty}$ which converges pointwise a.e.- $F$ to a monotone pure strategy limit $\sigma^{*}$. With more than one global bidder, however, we may no longer leverage better-reply security to conclude that $\sigma^{*}$ is an equilibrium.

Now augment the limit strategy profile $\sigma^{*}$ with a profile of tiebreaking strategies $\tau^{*}=$ $\left(\tau_{1}^{*}, \ldots, \tau_{N}^{*}\right)$ constructed as follows. For each strategy profile $\sigma^{k}$ along the sequence $\left\{\sigma^{k}\right\}_{k=1}^{\infty}$, let $\Gamma_{i l}^{k}\left(b_{l}\right) \equiv \operatorname{Pr}\left(\sigma_{i l}^{k}\left(Y_{i}\right) \leq b_{l}\right)$ denote the c.d.f. of bidder $i$ 's bid in auction $l, \bar{\Gamma}_{l}^{k}\left(b_{l}\right) \equiv$ $\prod_{i=1}^{N} \Gamma_{i l}^{k}\left(b_{l}\right)$ denote the c.d.f. of the maximum bid among all bidders in auction $l$, and 
$\bar{\gamma}_{l}^{k}\left(b_{l}\right)$ denote the (discrete) p.d.f. associated with the c.d.f $\bar{\Gamma}$. For each bidder $i$ and auction $l$, let $\tau_{i l}^{k}\left(y_{i}\right) \equiv \bar{\Gamma}_{l}^{k}\left(\sigma_{i l}^{k}\left(y_{i}\right)\right)-\frac{1}{2} \bar{\gamma}_{l}^{k}\left(\sigma_{i l}^{k}\left(y_{i}\right)\right)$ denote the c.d.f. of the maximum bid among all bidders in auction $l$, evaluated at the point $b_{l}=\sigma_{i l}^{k}\left(y_{i}\right)$, less $1 / 2$ the p.d.f. of the maximum bid among all bidders in auction $l$, also evaluated at the point $b_{l}=\sigma_{i l}^{k}\left(y_{i}\right)$.

Note the following properties of the function $\bar{\Gamma}_{l}^{k}\left(b_{l}\right)-\frac{1}{2} \bar{\gamma}_{l}^{k}\left(b_{l}\right)$. First, since each step of the c.d.f. $\bar{\Gamma}_{l}$ is equal to the p.d.f. $\bar{\gamma}_{l}^{k}\left(b_{l}\right), \bar{\Gamma}_{l}^{k}\left(b_{l}\right)-\frac{1}{2} \bar{\gamma}_{l}^{k}\left(b_{l}\right)$ is monotone in $b_{l}$. Second, consider any $k=1,2, \ldots$ and any two bids $b_{1}, b_{2} \in \ddot{B}_{l}^{k}$. Suppose WLOG that $b_{2} \geq b_{1}$. Clearly, if $b_{2}=b_{1}$, then $\bar{\Gamma}_{l}^{k}\left(b_{1}\right)-\frac{1}{2} \bar{\gamma}_{l}^{k}\left(b_{1}\right)=\bar{\Gamma}_{l}^{k}\left(b_{2}\right)-\frac{1}{2} \bar{\gamma}_{l}^{k}\left(b_{2}\right)$. Alternatively, if $b_{2}>b_{1}$, then

$$
\left[\bar{\Gamma}_{l}^{k}\left(b_{2}\right)-\frac{1}{2} \bar{\gamma}_{l}^{k}\left(b_{2}\right)\right]-\left[\bar{\Gamma}_{l}^{k}\left(b_{1}\right)-\frac{1}{2} \bar{\gamma}_{l}^{k}\left(b_{1}\right)\right]=\frac{1}{2}\left[\bar{\gamma}_{l}^{k}\left(b_{1}\right)+\bar{\gamma}_{l}^{k}\left(b_{2}\right)\right]+\bar{\Gamma}_{l}^{k}\left(\left(b_{1}, b_{2}\right)\right)
$$

where $\bar{\Gamma}_{l}^{k}\left(\left(b_{1}, b_{2}\right)\right)$ denotes the $\bar{\Gamma}_{l}^{k}$-measure of the open interval $\left(b_{1}, b_{2}\right)$.

Finally, for each bidder $i$, define an $L \times 1$ vector $\tau_{i}^{k}\left(y_{i}\right)=\left(\tau_{i 1}^{k}\left(y_{i}\right), \ldots, \tau_{i L}^{k}\left(y_{i}\right)\right)$ stacking up the functions $\tau_{i l}^{k}$ just defined. Observe that, by construction, $\tau_{1 l}^{k}, \ldots, \tau_{N l}^{k}$ preserve order of bids across bidders: i.e. for any bidders $i, j$, we have $\tau_{i l}^{k}\left(y_{i}\right) \gtreqless \tau_{j l}^{k}\left(y_{j}\right)$ as $\sigma_{i l}^{k}\left(y_{i}\right) \gtreqless \sigma_{j l}^{k}\left(y_{j}\right)$. Furthermore, the vector $\tau_{i}^{k}\left(y_{i}\right)$ inherits monotonicity of $\sigma_{i}^{k}: y_{i}^{\prime} \succeq y_{i}$ in the sense of $(P O)$ implies $\tau_{i}^{k}\left(y_{i}^{\prime}\right) \geq \tau_{i}^{k}\left(y_{i}\right)$ in the usual coordinatewise sense. Hence defining $\tau^{k}=\left(\tau_{1}^{k}, \ldots, \tau_{1}^{k}\right)$ and focusing on the subsequence such that $\sigma^{k} \rightarrow \sigma^{*}$, it follows that there exists a further subsequence $\left\{k_{j}\right\}_{j=1}^{\infty}$ such that $\tau^{k}$ also converges pointwise a.e.- $F$ to a monotone limit $\tau^{*}$.

What do we gain from this construction? The cardinal limit $\sigma^{*}$ cannot preserve ordinal information on bids along the sequence - a tie under $\sigma^{*}$ implies only that a positive measure of types have the same limit bid. But the ordinal limit $\tau^{*}$ does: if $\tau_{i l}^{*}\left(y_{i}\right)<\tau_{j l}^{*}\left(y_{j}\right)$, then we must eventually have $\tau_{i l}^{k}\left(y_{i}\right)<\tau_{j l}^{k}(y)$ and hence $\sigma_{i l}^{k}\left(y_{i}\right)<\sigma_{j l}^{k}\left(y_{j}\right)$ almost surely even if also $\sigma_{i l}^{k}\left(y_{i}\right) \rightarrow \sigma_{j l}^{k}\left(y_{j}\right)$ (and hence types $y_{i}, y_{j}$ tie under $\sigma^{*}$ ). Furthermore, the set of types experiencing any relevant tie under the limit profile $\tau^{*}$ will correspond, up to a set of measure zero, with the set of types eventually experiencing an equivalent tie along the sequence $\sigma^{k}$. These facts are central to our construction, so we state and prove them separately as a lemma.

Lemma 5. Consider any auction $l=1, \ldots, L$ and any $\left(\bar{b}_{l}, \bar{t}_{l}\right)$ such that there exists some bidder $i=1, \ldots, N$ and type $y_{i}$ such that $\sigma_{i l}^{k}\left(y_{i}\right) \rightarrow \bar{b}_{l}$ and $\tau_{l}^{k}\left(y_{i}\right) \rightarrow \bar{t}_{l}$ as $k \rightarrow 0$. Then for any bidder $j=1, \ldots, N$ and for any type $y_{j}$ of bidder $j$ such that $\lim _{k} \tau_{j l}^{k}\left(y_{j}\right)=\bar{t}_{l}$,

- If $\tau_{j l}^{*}\left(y_{j}\right)<\bar{t}_{l}$, then eventually $\sigma_{j l}^{k}\left(y_{j}\right)<\sigma_{i l}^{k}\left(y_{i}\right)$ as $k \rightarrow \infty$;

- If $\tau_{j l}^{*}\left(y_{j}\right)>\bar{t}_{l}$, then eventually $\sigma_{j l}^{k}\left(y_{j}\right)>\sigma_{i l}^{k}\left(y_{i}\right)$ as $k \rightarrow \infty$.

- If $\tau_{j l}^{*}\left(y_{j}\right)=\bar{t}_{l}$, then either (i) there exists $K<\infty$ such that $\sigma_{j l}^{k}\left(y_{j}\right)=\sigma_{i l}^{k}\left(y_{i}\right)$ for all $k>K$, or (ii) the $\bar{\Gamma}_{l}^{k}$-measure of the closed interval

$$
\left[\min \left\{\sigma_{i l}^{k}\left(y_{i}\right), \sigma_{j l}^{k}\left(y_{j}\right)\right\}, \max \left\{\sigma_{i l}^{k}\left(y_{i}\right), \sigma_{i l}^{k}\left(y_{i}\right)\right\}\right]
$$

approaches zero as $k \rightarrow \infty$. 
Further suppose that $\bar{t}_{l}>0$, and for each bidder $j$, let $\overline{\mathcal{Y}}_{j}$ be the set of types $y_{j} \in \mathcal{Y}_{j}$ such that $\sigma_{i l}^{k}\left(y_{i}\right) \rightarrow \bar{b}_{l}$ and $\tau_{i l}^{k}\left(y_{i}\right) \rightarrow \bar{t}_{l}$. Then there exists a sequence $\left\{b_{l}^{k}\right\}_{k=1}^{k}$, with $b_{l}^{k} \in \ddot{\mathcal{B}}_{l}^{k}$ for each $k=1,2, \ldots$ and $b_{l}^{k} \rightarrow \bar{b}_{l}$, such that for all bidders $j$, the $F_{j}$-measure of types $y_{j}$ such that $y_{j} \in \overline{\mathcal{Y}}_{j}$ but $\sigma_{j l}^{k}\left(y_{j}\right) \neq b_{l}^{k}$ converges to zero as $k \rightarrow \infty$.

Proof of Lemma 5. First suppose that $\tau_{j l}^{*}\left(y_{j}\right)<\bar{t}_{l}$. Consider the subset of types $y_{j}$ such that both $\sigma_{j l}^{k}\left(y_{j}\right)$ and $\tau_{j l}^{k}\left(y_{j}\right)$ are pointwise convergent; recall that this set has full measure with respect to $F_{j}$. If $\tau_{j l}^{*}\left(y_{j}\right)<\bar{t}_{l}$, then by definition

$$
\lim \left\{\bar{\Gamma}_{l}^{k}\left(\sigma_{j l}^{k}\left(y_{j}\right)\right)-\frac{1}{2} \bar{\gamma}_{l}^{k}\left(\sigma_{j l}^{k}\left(y_{j}\right)\right)\right\}<\bar{t}_{l}=\lim \left\{\bar{\Gamma}_{l}^{k}\left(\sigma_{i l}^{k}\left(y_{i}\right)\right)-\frac{1}{2} \bar{\gamma}_{l}^{k}\left(\sigma_{i l}^{k}\left(y_{i}\right)\right)\right\} .
$$

But from above, this can hold only if eventually $\sigma_{j l}^{k}\left(y_{j}\right)<\sigma_{i l}^{k}\left(y_{i}\right)$.

Now suppose that that $\tau_{j l}^{*}\left(y_{j}\right)>\bar{t}_{l}$. In this case, reversing the arguments in the last paragraph shows that we must eventually have $\sigma_{j l}^{k}\left(y_{j}\right)>\sigma_{i l}^{k}\left(y_{i}\right)$.

Next suppose that both $\tau_{j l}^{k}\left(y_{j}\right) \rightarrow \bar{t}_{l}$ and $\tau_{i l}^{k}\left(y_{i}\right) \rightarrow \bar{t}_{l}$. Further suppose that there does not exist any $K<\infty$ such that $k>K$ implies $\sigma_{i l}^{k}\left(y_{i}\right)=\sigma_{j l}^{k}\left(y_{j}\right)$ for all $k>K$. Letting $b_{1}^{k} \equiv \min \left\{\sigma_{i l}^{k}\left(y_{i}\right), \sigma_{j l}^{k}\left(y_{j}\right)\right\}$ and $b_{2}^{k} \equiv \max \left\{\sigma_{i l}^{k}\left(y_{i}\right), \sigma_{j l}^{k}\left(y_{j}\right)\right\}$, we must then have $b_{1}^{k}<b_{2}^{k}$, at least along a subsequence. But recall from above that $b_{1}^{k}<b_{2}^{k}$ implies

$$
\begin{aligned}
{\left[\bar{\Gamma}_{l}^{k}\left(b_{2}^{k}\right)-\frac{1}{2} \bar{\gamma}_{l}^{k}\left(b_{2}^{k}\right)\right]-\left[\bar{\Gamma}_{l}^{k}\left(b_{1}^{k}\right)-\frac{1}{2} \bar{\gamma}_{l}^{k}\left(b_{1}^{k}\right)\right] } & =\frac{1}{2}\left[\bar{\gamma}_{l}^{k}\left(b_{1}^{k}\right)+\bar{\gamma}_{l}^{k}\left(b_{1}^{k}\right)\right]+\bar{\Gamma}_{l}^{k}\left(\left(b_{1}^{k}, b_{2}^{k}\right)\right) \\
& \geq \frac{1}{2}\left[\bar{\gamma}_{l}^{k}\left(b_{1}^{k}\right)+\bar{\gamma}_{l}^{k}\left(b_{1}^{k}\right)+\bar{\Gamma}_{l}^{k}\left(\left(b_{1}^{k}, b_{2}^{k}\right)\right)\right] \\
& =\bar{\Gamma}_{l}^{k}\left(\left[b_{1}^{k}, b_{2}^{k}\right]\right) .
\end{aligned}
$$

The left-hand side of this inequality is identically $\left|\tau_{j l}^{k}\left(y_{j}\right)-\tau_{i l}^{k}\left(y_{i}\right)\right|$, which approaches zero by hypothesis. Meanwhile, the right-hand side is the $\bar{\Gamma}_{l}^{k}$-measure of the closed interval $\left[b_{1}^{k}, b_{2}^{k}\right]$. Hence if $\tau_{j l}^{k}\left(y_{j}\right) \rightarrow \bar{t}_{l}, \tau_{i l}^{k}\left(y_{i}\right) \rightarrow \bar{t}_{l}$, and there does not exist a $K<\infty$ such that $\sigma_{i l}^{k}\left(y_{i}\right)=\sigma_{j l}^{k}\left(y_{j}\right)$ for all $k>K$, then $\bar{\Gamma}_{l}^{k}\left(\left[b_{1}^{k}, b_{2}^{k}\right]\right) \rightarrow 0$. This establishes the claim.

Lastly, consider the final statement of Lemma 5 . Suppose that $\bar{t}_{l}>0$, and let $\overline{\mathcal{Y}}_{j}$ denote the set of types $y_{j} \in \mathcal{Y}_{j}$ such that $\sigma_{j l}^{k}\left(y_{j}\right) \rightarrow \bar{b}_{l}$ and $\tau_{j l}^{k}\left(y_{j}\right) \rightarrow \bar{t}_{l}$; note that this set coincides up to a set of $F_{j}$-measure 0 with the set of types $y_{j}$ such that $\tau_{j l}^{*}\left(y_{j}\right)=\bar{t}_{l}$.

Without loss, restrict attention to $j$ such that the $F_{j}$-measure of $\overline{\mathcal{Y}}_{j}$ strictly exceeds zero; the statement clearly holds trivially otherwise.

For each $k$, let $\underline{b}_{l}^{k}$ and $\bar{b}_{l}^{k}$ be the minimum and maximum, respectively, among bids $\sigma_{j l}^{k}\left(y_{j}\right)$ submitted by some type $y_{j} \in \overline{\mathcal{Y}}_{j}$ of some bidder $j$, and let $b_{l}^{k}$ be the conditional $\bar{\Gamma}_{l}^{k}$-median of the interval $\left[\underline{b}_{l}^{k}, \bar{b}_{l}^{k}\right]$ (rounded up within $\ddot{\mathcal{B}}_{l}^{k}$ as necessary). Note that this sequence is well defined since at least one bidder $j$ submits bids in the interval $\left[\underline{b}_{l}^{k}, \bar{b}_{l}^{k}\right]$ with positive probability, and since $\bar{t}_{l}>0$ implies that for sufficiently large $k$ this bidder is the maximum bidder with strictly positive probability.

With this sequence $\left\{b_{l}^{k}\right\}_{k=1}^{\infty}$ in hand, consider bidder $j$, and let $\mathcal{Y}_{j}^{k,-}\left(b_{l}^{k}\right), \mathcal{Y}_{j}^{k,=}\left(b_{l}^{k}\right)$, and 
$\mathcal{Y}_{j}^{k,+}\left(b_{l}^{k}\right)$ be subsets of $\overline{\mathcal{Y}}_{j}$ defined as follows:

$$
\begin{aligned}
& \mathcal{Y}_{j}^{k,-}\left(b_{l}^{k}\right)=\left\{y_{j} \in \overline{\mathcal{Y}}_{j} \mid \sigma_{j l}^{k}\left(y_{j}\right)<b_{l}^{k}\right\}, \\
& \mathcal{Y}_{j}^{k,=}\left(b_{l}^{k}\right)=\left\{y_{j} \in \overline{\mathcal{Y}}_{j} \mid \sigma_{j l}^{k}\left(y_{j}\right)=b_{l}^{k}\right\}, \\
& \mathcal{Y}_{j}^{k,+}\left(b_{l}^{k}\right)=\left\{y_{j} \in \overline{\mathcal{Y}}_{j} \mid \sigma_{j l}^{k}\left(y_{j}\right)>b_{l}^{k}\right\} .
\end{aligned}
$$

For any (measurable) $S \subset \mathcal{Y}_{j}$, let $F_{j}(S)$ denote the $F_{j}$-measure of $S$. Note that for each $k$ we have

$$
F_{j}\left(\overline{\mathcal{Y}}_{j}\right)=F_{j}\left(\mathcal{Y}_{j}^{k,-}\left(b_{l}^{k}\right)\right)+F_{j}\left(\mathcal{Y}_{j}^{k,=}\left(b_{l}^{k}\right)\right)+F_{j}\left(\mathcal{Y}_{j}^{k,+}\left(b_{l}^{k}\right)\right) .
$$

First consider $F_{j}\left(\mathcal{Y}_{j}^{k,-}\left(b_{l}^{k}\right)\right)$, and suppose that $\lim _{k \rightarrow \infty} F_{j}\left(\mathcal{Y}_{j}^{k,-}\left(b_{l}^{k}\right)\right) \neq 0$. Since $F_{j}\left(\mathcal{Y}_{j}^{k,-}\left(b_{l}^{k}\right)\right)$ is nonnegative, there must then exist some $\epsilon>0$ such that, at least along a subsequence, we have $F_{j}\left(\mathcal{Y}_{j}^{k,-}\left(b_{l}^{k}\right)\right) \geq \epsilon$. Pass to this subsequence as necessary.

Now, at each iteration $k$, let $\tilde{b}_{l}^{k}$ denote the median bid submitted by types $y_{j}$ within the subset $\mathcal{Y}_{j}^{k,-}\left(b_{l}^{k}\right)$ (rounding down in case of ties). Note that this is again well defined since by hypothesis $F_{j}\left(\mathcal{Y}_{j}^{k,-}\left(b_{l}^{k}\right)\right) \geq \epsilon$. Furthermore, by construction, at least $F_{j}$-measure $\epsilon / 2$ of types $\left.y_{j} \in \mathcal{Y}_{j}^{k,-}\left(b_{l}^{k}\right)\right)$ have $\sigma_{j l}^{k}\left(y_{j}\right) \leq \tilde{b}_{l}^{k}$, and at least $F_{j}$-measure $\epsilon / 2$ of types $\left.y_{j} \in \mathcal{Y}_{j}^{k,-}\left(b_{l}^{k}\right)\right)$ have $\sigma_{j l}^{k}\left(y_{j}\right) \geq \tilde{b}_{l}^{k}$ (although these sets could overlap).

Next consider the c.d.f. of the maximum bid at step $k$, evaluated at $\tilde{b}^{k}$. By definition,

$$
\bar{\Gamma}_{l}^{k}\left(\tilde{b}_{l}^{k}\right)=\Gamma_{j l}^{k}\left(\tilde{b}_{l}^{k}\right) \prod_{i \neq j} \Gamma_{i l}^{k}\left(\tilde{b}^{k}\right) .
$$

Since $\bar{\Gamma}_{l}^{k}\left(\tilde{b}_{l}^{k}\right) \geq \bar{\Gamma}_{l}^{k}\left(\tilde{b}_{l}^{k}\right)-\frac{1}{2} \bar{\gamma}_{l}^{k}\left(\tilde{b}_{l}^{k}\right) \rightarrow \bar{t}_{l}>0$, we must eventually have $\bar{\Gamma}_{l}^{k}\left(\tilde{b}_{l}^{k}\right)>\tilde{t}_{l}$ for some $\tilde{t}_{l}>0$, and hence $\prod_{i \neq j} \Gamma_{i l}^{k}\left(\tilde{b}_{l}^{k}\right)>\tilde{t}_{l}$ since $\Gamma_{j l}^{k}\left(\tilde{b}_{l}^{k}\right) \leq 1$. Pass to this subsequence as needed.

Finally, consider any $y_{1} \in \mathcal{Y}_{j}^{k,=}\left(b_{l}^{k}\right) \cup \mathcal{Y}_{j}^{k,+}\left(b_{l}^{k}\right)$ and any $y_{2}$ such that $\sigma_{j l}^{k}\left(y_{2}\right) \leq \tilde{b}_{l}^{k}$. Observe that

$$
\begin{aligned}
\tau_{j l}^{k}\left(y_{1}\right) & =\bar{\Gamma}_{l}^{k}\left(b_{l}^{k}\right)-\frac{1}{2} \bar{\gamma}_{l}^{k}\left(b_{l}^{k}\right) \\
& \geq \bar{\Gamma}_{l}^{k}\left(\left[0, \tilde{b}_{l}^{k}\right)\right)+\bar{\gamma}_{l}^{k}\left(\tilde{b}_{l}^{k}\right)+\bar{\Gamma}_{l}^{k}\left(\left(\tilde{b}_{l}^{k}, b^{k}\right)\right) \\
& \geq \bar{\Gamma}_{l}^{k}\left(\left[0, \tilde{b}_{l}^{k}\right)\right)+\frac{1}{2} \bar{\gamma}_{l}^{k}\left(\tilde{b}_{l}^{k}\right)+\frac{1}{2}\left[\bar{\gamma}_{l}^{k}\left(\tilde{b}_{l}^{k}\right)+\bar{\Gamma}_{l}^{k}\left(\left(\tilde{b}_{l}^{k}, b_{l}^{k}\right)\right)\right] \\
& =\bar{\Gamma}_{l}^{k}\left(\tilde{b}_{l}^{k}\right)-\frac{1}{2} \bar{\gamma}_{l}^{k}\left(\tilde{b}_{l}^{k}\right)+\frac{1}{2} \bar{\Gamma}_{l}^{k}\left(\left[\tilde{b}_{l}^{k}, b_{l}^{k}\right)\right) \\
& \geq \tau_{j l}^{k}\left(y_{2}\right)+\frac{1}{2} \bar{\Gamma}_{l}^{k}\left(\left[\tilde{b}_{l}^{k}, b_{l}^{k}\right)\right) .
\end{aligned}
$$

By definition, $\bar{\Gamma}_{l}^{k}\left(\left[\tilde{b}_{l}^{k}, b_{l}^{k}\right)\right)$ is the probability that the maximum bid in step $k$ falls in the interval $\left[\tilde{b}_{l}^{k}, b_{l}^{k}\right)$. One way (of many) this can occur is that bidder $j$ submits a bid in $\left[\tilde{b}_{l}^{k}, b_{l}^{k}\right)$, while all other bidders submit bids weakly less than $\tilde{b}_{l}^{k}$. Since, by construction, $j$ submits 
a bid in the interval $\left[\tilde{b}_{l}^{k}, b_{l}^{k}\right)$ with probability at least $\epsilon / 2$, it follows that

$$
\bar{\Gamma}_{l}^{k}\left(\left[\tilde{b}_{l}^{k}, b_{l}^{k}\right)\right) \geq \operatorname{Pr}\left(\sigma_{j l}^{k} \in\left[\tilde{b}_{l}^{k}, b_{l}^{k}\right)\right) \prod_{i \neq j} \Gamma_{i l}^{k}\left(\tilde{b}_{l}^{k}\right) \geq \frac{\epsilon \tilde{t}_{l}}{2} .
$$

Hence, for all $k$, for all $y_{1} \in \mathcal{Y}_{j}^{k,=}\left(b_{l}^{k}\right) \cup \mathcal{Y}_{j}^{k,+}\left(b_{l}^{k}\right)$, and for all $y_{2} \in \mathcal{Y}_{j}\left(\bar{t}_{l}\right)$ such that $\sigma_{j l}^{k}\left(y_{2}\right) \leq$ $\tilde{b}_{l}^{k}$, we must have

$$
\tau_{j l}^{k}\left(y_{1}\right) \geq \tau_{j l}^{k}\left(y_{2}\right)+\frac{\epsilon \tilde{t}_{l}}{4}>0 .
$$

But recall that, for each $k$, the $F_{j}$-measure of the set $\left\{y_{j} \in \mathcal{Y}_{j}^{k,-}: \sigma_{j l}^{k}\left(y_{j}\right) \leq \tilde{b}_{l}^{k}\right\}$ is at least $\epsilon / 2>0$, while the $F_{j}$-measure of the set $\mathcal{Y}_{j}^{k,=}\left(b_{l}^{k}\right) \cup \mathcal{Y}_{j}^{k,+}\left(b_{l}^{k}\right)$ is at least $F_{j}\left(\overline{\mathcal{Y}}_{j}\right) / 2>0$. In other words, we can find two subsets of $\overline{\mathcal{Y}}_{j}$ of $F_{j}$-measure bounded away from zero, such that for every $k$, all values of $\tau_{j l}^{k}(\cdot)$ on one subset are bounded away from all values of $\tau_{j l}^{k}(\cdot)$ on the other subset by a strictly positive distance. In this case, if $\tau_{j l}^{k}\left(y_{j}\right) \rightarrow \bar{t}_{l}$ on one subset, we cannot have $\tau_{j l}^{k}\left(y_{j}\right) \rightarrow \bar{t}_{l}$ on the other, contradicting the hypothesis that $\tau_{j l}^{k}\left(y_{j}\right) \rightarrow \bar{t}_{l}$ for all $y_{j} \in \overline{\mathcal{Y}}_{j}$. It follows that we must have $\lim _{k \rightarrow \infty} F_{j}\left(\mathcal{Y}_{j}^{k,-}\left(b_{l}^{k}\right)\right)=0$.

To complete the argument, consider $F_{j}\left(\mathcal{Y}_{j}^{k,+}\left(b_{l}^{k}\right)\right)$, and suppose that $\lim _{k \rightarrow \infty} F_{j}\left(\mathcal{Y}_{j}^{k,+}\left(b_{l}^{k}\right)\right) \neq$ 0 . In this case, taking $\tilde{b}_{l}^{k}$ to be the median bid submitted by types $y_{j} \in \mathcal{Y}_{j}^{k,+}\left(b_{l}^{k}\right)$, and focusing on the limit of $\tau_{j l}^{k}(\cdot)$ among types whose bids weakly exceed $\tilde{b}_{l}^{k}$, we find that the hypothesis $\lim _{k \rightarrow \infty} F_{j}\left(\mathcal{Y}_{j}^{k,+}\left(b_{l}^{k}\right)\right) \neq 0$ implies a contradiction analogous to the one above. Hence we must also have $F_{j}\left(\mathcal{Y}_{j}^{k,+}\left(b_{l}^{k}\right)\right) \rightarrow 0$. This establishes the claim.

Lemma 5 immediately implies that bidder $i$ with type $y_{i}$ cannot strictly gain from submitting any $(\bar{b}, \bar{t})$ which is on the equilibrium path in the sense that for each auction $l$, there exists some bidder $j=1, \ldots, N$ and type $y_{j}$ with $\sigma_{i l}^{k}\left(y_{i}\right) \rightarrow \bar{b}_{l}$ and $\bar{\Gamma}_{l}^{k}\left(\sigma_{i l}^{k}\left(y_{i}\right)\right) \rightarrow \bar{t}_{l}$. For by Lemma 5 , the outcomes generated by such a $(\bar{b}, \bar{t})$ can differ on at most a set of measure approaching zero from the limiting outcomes which would have eventually obtained had bidder $i$ imitated the relevant type $y_{j}$ of bidder $j$ in auction $l$ along the sequence of equilibria $\sigma^{k}$. Hence if deviating to $(\bar{b}, \bar{t})$ is strictly profitable under $\sigma^{*} \times \tau^{*}, i$ must eventually also have had a strictly profitable deviation at some point along the sequence $\sigma^{k}$. This contradicts the hypothesis that $\sigma^{k}$ is a sequence of equilibria.

So $i$ cannot have a profitable deviation which is on the equilibrium path in the sense above. To ensure that the profile $\sigma^{*} \times \tau^{*}$ is an equilibrium, it is therefore sufficient to construct a collection of tiebreaking precendence rules $\rho_{1}^{*}, \ldots, \rho_{L}^{*}$ such that the set of deviations available to $i$ under $\sigma^{*} \times \tau^{*}$ are payoff equivalent to those on the equilibrium path.

Toward this end, consider the tiebreaking rule $\rho_{l}^{*}(t)$ equal to the c.d.f. of the random variable $T=\max \left\{\tau_{1 l}^{*}\left(Y_{1}\right), \ldots, \tau_{N l}^{*}\left(Y_{N}\right)\right\}$ evaluated at $t$. Then $\rho_{l}^{*}$ is a weakly monotone function from $[0,1]$ to $[0,1]$. Furthermore, if the auctioneer evaluates ties according to the tiebreaking rule $\rho_{l}^{*}(t)$, then by construction submitting any tiebreaking signal $t^{*} \in$ $[0,1]$ is equivalent (up to an irrelevant set of measure zero) to submitting the next lowest 
tiebreaking signal on the equilibrium path. Hence under tiebreaking rule $\rho_{l}^{*}$, bidder $i$ 's set of feasible deviations in auction $l$ is payoff equivalent to her set of feasible deviations on the equilibrium path. In view of the arguments above, it follows that $\sigma^{*} \times \tau^{*}$ is an equilibrium under the $L \times 1$ vector of tiebreaking rules $\rho^{*}=\left(\rho_{1}^{*}, \ldots, \rho_{L}^{*}\right)$.

\section{Example of a simultaneous standard auction game with mul- tiple global bidders that is not better-reply secure.}

Example 4. Consider a setting where two symmetric bidders $i=1,2$ compete in two first-price auctions, with reserve price $r=1$ in auction 1 and $r=0$ in auction 2. Each bidder $i$ has standalone valuations $Y_{i}^{1,0}$ and $Y_{i}^{0,1}$ uniformly distributed on $[0,1]$, and a combinatorial valuation $Y_{i}^{1,1}=Y_{i}^{0,1}+e_{i}^{1,1}$ with $e_{i}^{1,1}$ continuously distributed on [1,2], with $\left(Y_{i}^{0,1}, Y_{i}^{1,0}, e_{i}^{1,1}\right)$ mutually independent. In what follows, let $F\left(y^{1,1}\right)$ denote the marginal c.d.f of the combinatorial valuation $Y_{i}^{1,1}$.

In this setting, consider the following sequence of bidding strategies $n=1,2,3, \ldots$ In auction 1 , bidders bid according to the strategy $\beta_{1}^{n}\left(y_{i}\right)=1+F\left(y_{i}^{1,1}\right) / n$. Meanwhile, in auction 2 , each bidder $i$ bids according to the fixed strategy $\beta_{2}\left(y_{i}\right)=B\left(y_{i}^{1,1}\right)-1$, where $B(\cdot)$ is the strategy describing a symmetric Bayesian Nash equilibrium of a standard first-price auction with standalone valuations drawn from distribution $F(\cdot)$ : i.e.,

$$
B(v)=v-\int_{1}^{v} \frac{F(z)}{F(v)} d z
$$

In other words, in auction 1 , both bidders submit strategies converging to the reserve price $r=1$. Meanwhile, in auction 2, they bid "as if" competing in a first-price auction in which both bidders draw standalone valuations according to $V_{i}=Y_{i}^{1,1}-1$.

Note the following features of this sequence of strategies. First, for each bidder, bids in each auction are comonotone in $y^{1,1}$, which implies that each bidder either wins both auctions, or wins neither. Second, the bidder who wins both auctions is the one with the highest valuation for the combination. Third, along the sequence, bids in auction 1 are monotonically decreasing toward the reserve price $r=1$, while expected payments in auction 2 are constant. Hence, as $n \rightarrow \infty$, payoffs for both bidders are monotonically increasing toward the limit

$$
\bar{\pi}=\int_{1}^{3}\left[y^{1,1}-B\left(y^{1,1}\right)\right] F\left(y^{1,1}\right) d F\left(y^{1,1}\right),
$$

which also equals the payoff which each bidder would obtain if they were instead competing in a standard first-price auction for a single object with valuations drawn from symmetric distribution $F$.

Let $\bar{\beta}=\lim _{n \rightarrow \infty}\left(\beta_{1}^{n}, \beta_{2}^{n}\right)$ be the limit of this sequence of strategy profiles as $n \rightarrow \infty$ : i.e. $\bar{\beta}_{1}\left(y_{i}\right)=1, \bar{\beta}_{2}(y)=\beta_{1}^{n}\left(y_{i}\right)=B\left(y_{i}^{1,1}\right)-1$. Clearly, this limit strategy profile $\bar{\beta}$ will involve a tie at $b_{11}=b_{21}=1$ in auction 1 with certainty. Furthermore, the limit strategy 
profile $\bar{\beta}$ will not be an equilibrium. ${ }^{20}$

Building on this example, we aim to show two further results. First, the game is not continuously secure in the sense of Barelli and Meneghel (2013), which implies that it is not multiply secure in the sense of McLennan, Monteiro and Tourky (2013) or better-reply secure in the sense of Reny (1999). Second, while $\bar{\beta}$ is not a Bayesian Nash equilibrium with independent tiebreaking, it is part of an equilibrium with endogenous tiebreaking.

We begin with the second claim: $\bar{\beta}$ is an equilibrium bidding strategy with endogenous tiebreaking. To see this, suppose we augment the strategy space of the auction game by allowing each bidder $i$ to submit, in addition to their bid $b_{i j}$, a cheap-talk signal $t_{i j} \in[0,1]$ used by the auctioneer to break ties in auction $j=\{1,2\}$. Specifically, suppose that, in the event that $b_{1 j}=b_{2 j}$, the auctioneer awards object $j$ to the bidder submitting the highest tiebreaking signal in auction $j$ (allocating randomly in the event that tiebreaking signals are also tied). With this simple extension, $\bar{\beta}$ is in fact an equilibrium bidding strategy.

Toward this end, augment the limit bid strategy $\bar{\beta}$ with a signaling strategy such that bidder $i$ submits tiebreaking signal $t_{i 1}=\phi\left(y_{i}^{1,1}\right)$ in auction 1 , where $\phi(\cdot)$ is any strictly monotone function from $\mathbb{R}$ to $[0,1] .{ }^{21}$ The specific function $\phi$ considered is inessential; for our purposes, all that matters is that both players employ the same signaling strategy, and that this signaling strategy is a strictly monotone function of (only) the joint valuation $Y_{i}^{1,1}$. Furthermore, since the limit profile $\bar{\beta}$ almost never yields ties in auction 2, any profile of signaling strategies in auction 2 will be consistent with equilibrium. We aim to show that, taking rival bidding and signaling strategies as given, neither bidder can gain by changing either their signal or their bid.

First note that both bidders submit the minimum bid in auction 1 . Hence any profitable deviation must involve changing either the signaling strategy in auction 1 or the bidding strategy in auction 2 .

Next, holding bid $b_{1}=1$ fixed, suppose that bidder $i$ submits bid $B(z)-1$ in auction 2 (for $z$ not necessarily equal to $y_{i}^{1,1}$; as usual, we can express any potentially profitable deviation in this way). Then it is also optimal for bidder $i$ to submit tiebreaking signal $\phi(z)$ in auction 1 . For conditional on winning auction 2, each bidder always prefers to win auction 1 also: $Y^{1,1}-Y^{0,1}$ always exceeds 1 . Furthermore, neither bidder wants to win auction 1 without also winning auction 2: $Y^{1,0}<1$ almost surely. Setting $t_{i 1}>\phi(z)$ would induce events in which bidder $i$ wins auction 1 but not auction 2 ; setting $t_{i 1}<\phi(z)$ would induce events in which $i$ wins auction 2 but not auction 1 . In either case, bidder $i$ could at least weakly, and almost always strictly, gain from submitting signal $t_{i 1}=\phi(z)$ instead.

In other words, we can restrict attention to deviations of the form $(1, B(z)-1, \phi(z))$ for some $z \in[1,3]$. By construction, at any such candidate deviation, bidder $i$ either wins both auctions or wins neither, paying total bid $B(z)$ and receiving joint valuation $y_{i}^{1,1}$ in the

\footnotetext{
${ }^{20}$ Formally, with independent tiebreaking, each bidder $i$ bidding $b_{i j}=1$ will win auction 1 with probability $1 / 2$ under the limit strategies. Hence, in the events in which they win auction 2 , each bidder $i$ will receive $y_{i}^{1,1}$ with probability $1 / 2$, and $y_{i}^{0,1}<y_{i}^{1,1}$ with probability $1 / 2$, rather than receiving $y_{i}^{1,1}$ with certainty as along the sequence. Consequently, the limit bidding strategy for auction 2, which relies on both bidders knowing that in the events they win auction 2 they will also win auction 1, will not be an equilibrium.

${ }^{21}$ For concreteness, one could for instance normalize $\phi\left(y_{i}^{1,1}\right)=F\left(y_{i}^{1,1}\right)$
} 
event of a win. Furthermore, given that their rival is following the equilibrium strategies specified above, bidder $i$ submitting deviation bid $(1, B(z)-1, \phi(z))$ wins both auctions with probability $F(z)$. Bidder $i$ 's problem is therefore to choose $z \in[1,3]$ to solve

$$
\max _{z}\left(y_{i}^{1,1}-1-(B(z)-1)\right) F(z)=\max _{z}\left(y_{i}^{1,1}-B(z)\right) F(z) .
$$

But this problem is formally equivalent to the bidding problem in a first-price auction in which both bidders draw effective valuations $y_{i}^{1,1}$ from $F(\cdot)$. By construction, $B(z)$ is the equilibrium bidding strategy corresponding to such an auction, which implies that each bidder $i$ 's optimal choice is $z=y_{i}^{1,1}$. Hence a bidder with type $y_{i}^{1,1}$ optimally submits bid vector $\left(1, B\left(y_{i}^{1,1}\right)-1\right)$ and tiebreaking signal $\tau\left(y_{i}^{1,1}\right)$. Note that, by construction, each bidder's payoff under this equilibrium is equal to the limit payoff $\bar{\pi}$ defined above.

We now return to the question of whether the simultaneous auction game is payoff secure. Toward this end, we consider the related definitions of Barelli and Meneghel (2013), McLennan, Monteiro and Tourky (2011), and Reny (1999). Intuitively, adapted to our context, these papers define a game as payoff secure at strategy profile $\bar{\beta}$ if, in some neighborhood $\mathcal{B}$ of $\bar{\beta}$, the following two conditions hold: (i) every player $i$ can secure a payoff above some threshold $\tilde{\pi}_{i}$ against every rival strategy contained in $\mathcal{B}$, and (ii) at each possible strategy profile $\tilde{\beta}$ in $\mathcal{B}$, some bidder is earning a payoff $\pi_{i}(\tilde{\beta})$ strictly below the payoff $\tilde{\pi}_{i}$ which they are able to secure. The precise definition of "secure a payoff" varies across studies, with the continuous security concept of Barelli and Meneghel (2013) nesting the multiple security concept of McLennan, Monteiro and Tourky (2011), which nests the better-reply security concept of Reny (1999). Essentially, however, all three definitions require each bidder $i$ to be able to use some strategy or strategy correspondence to guarantee a payoff above the threshold $\tilde{\pi}_{i}$ against any rival strategy in the neighborhood $\mathcal{B}$, where the securing strategies can in principle depend on which rival strategies are played at each point in the neighborhood.

For our purposes, it is sufficient to demonstrate that, against rival strategy $\beta_{n}$, any own strategy must yield an expected payoff strictly below the limit payoff $\bar{\pi}$; this implies that it is impossible for any bidder to secure a payoff equal to or greater than $\bar{\pi}_{i}$ using any strategy or combination of strategies. Toward this end, suppose to the contrary that there exists a strategy $\tilde{\beta}$ for bidder $i$ yielding expected payoff weakly exceeding $\bar{\pi}$ when the rival bidder follows strategy $\beta_{n}$. Observe that, by following strategy $\tilde{\beta}_{1}(\cdot)$ in auction 1 , bidder $i$ with type $y_{i}$ wins auction 1 against all rival types $y_{j}$ such that

$$
y_{j}^{1,1} \leq F^{-1}\left(n \tilde{\beta}_{1}\left(y_{i}\right)-n\right)
$$

Now return to the equilibrium with tiebreaking developed above. Observe that, by submitting bid vector $\left(1, \tilde{\beta}_{2}\right)$ and submitting tiebreaking signal $\phi\left(F^{-1}\left(n \tilde{\beta}_{1}\left(y_{i}\right)-n\right)\right)$, bidder $i$ could achieve the same ex-post allocations and auction- 2 payments as she achieves by playing $\tilde{\beta}$ against $\beta_{n}$. But from above, we know that the expected payoff from such a deviation must be at least weakly below $\bar{\pi}$, since $\bar{\pi}$ is the payoff from bidder $i$ 's optimal play given the strategy of her rival. It follows that we must have bidder $i$ 's expected payoff from playing $\tilde{\beta}$ against $\beta_{n}$ at least weakly below $\bar{\pi}$. Furthermore, since $\beta_{n}(\cdot)>1$ almost 
surely, the strategy $\tilde{\beta}$ must involve either losing auction 1 almost surely, or an average bid in auction 1 which strictly exceeds 1 . Either would strictly reduce payoffs relative to the limiting payoff $\bar{\pi}$. Hence the inequality in expected payoffs must be strict.

Since any strategy played against $\beta_{n}$ must yield a payoff strictly below $\bar{\pi}$, we conclude that any payoff $\tilde{\pi}$ securable by $i$, using any strategy or strategy mapping allowable under the definitions above, must be strictly below $\bar{\pi}$. Furthermore, for any possible $\tilde{\pi}<\bar{\pi}$, we have exhibited a sequence of strategies which are contained in any neighborhood of $\bar{\beta}$, such that both bidders eventually obtain payoffs strictly greater than $\tilde{\pi}$. In other words, for any payoff $\tilde{\pi}$ which bidders could potentially secure, and in every neighborhood of the limit strategy $\bar{\beta}$, there exists a strategy profile $\beta_{m}(\cdot)$ such that both bidders earn payoffs strictly greater than $\tilde{\pi}$. We conclude that conditions (i) and (ii) of the payoff security definition cannot hold simultaneously. Hence the game is not payoff secure.

Finally, we briefly discuss the analyses of Bagh and Jofre (2006) and Carmona (2009), which also concern extensions to better-reply security but are not nested by Barelli and Meneghel (2013). Bagh and Jofre (2006) provide weaker sufficient conditions than Reny (1999) under which better reply security (as defined by Reny 1999) holds. Since we have already shown directly that our game is not better reply secure, these conditions are unfortunately not helpful here. Meanwhile, Carmona (2009) shows that weak better reply security plus weak upper semicontinuity are sufficient for existence of Nash equilibrium. Weak upper semicontinuity requires that for each $(\tilde{\beta}, \tilde{\pi})$ pair in the frontier of the closure of the function mapping strategies to payoffs, there exists at least one player $i$ who can earn a payoff strictly greater than $\pi_{i}$ by deviating to some alternative strategy $\beta_{i}$. But as shown above, the strategy-payoff pair $(\bar{\beta}, \bar{\pi})$ is in the frontier of the closure of the payoff function, and no bidder $i$ can earn a payoff above $\bar{\pi}_{i}$ if their rival bids according to $\bar{\beta}$. 